\title{
Lagrangian Analysis led design of a Shock Recovery Plate Impact Experiment
}

Rade Vignjevic $^{1}$, Kevin Hughes ${ }^{1}$, Tom De Vuyst ${ }^{1}{ }^{*}$, Nenad Djordjevic $^{1}$, James

Campbell $^{1}$, Miroslav Stojkovic ${ }^{1}$, Omkar Gulavani ${ }^{1}$, Stefan Hiermaier ${ }^{2}$

1 - Crashworthiness, Impacts and Structural Mechanics Group,

School of Engineering, Cranfield University, BEDS, MK43 0AL, UK

$\underline{\text { www.cranfield.ac.uk/soe/structures }}$

$\stackrel{2}{2}$ - Deputy Director, Ernst-Mach Institut (EMI), Eckerstraße 4, 79104 Freiburg,

Germany

* - Corresponding author: Tel: +44 1234758286

Email addresses:

v.rade@cranfield.ac.uk; k.hughes@cranfield.ac.uk; t.devuyst@cranfield.ac.uk;

n.djordjevic@cranfield.ac.uk;j.c.campbell@cranfield.ac.uk ;

o.gulavani@cranfield.a.c.uk; hiermaier@emi.fraunhofer.de

Keywords: Flyer plate; Shock recovery; Momentum trap; distance-time diagrams;

\begin{abstract}
Shock recovery techniques, such as the flyer-plate impact test, are used to examine a material that has been subjected to a single well-defined shock, followed by a single release wave. One of the key requirements of this type of technique is that the process should be such that any change found in the sample after recovery, can only be attributed to the shock process alone. Therefore, the principal problem for a test specimen-fixture assembly is that it is designed such that the loading history of the recovered specimen is known. This has motivated this research through the analysis
\end{abstract}


led design of a shock recovery experiment. The choice of Lagrangian Finite Element Analysis for this design work was driven by the method's ability to accurately track history variables (for plastic deformation) and treat contact interactions which are crucial in this problem.

Starting from an initial configuration, LS-Dyna has been used to analyse in detail the resulting wave propagation to ensure the generation of a uniaxial strain state in the specimen through Lagrangian distance-time diagrams. These iso-maps enabled the identification of potential shortcomings with the initial design, in terms of the transmission of contact and the influence of radial release waves at the different boundaries between specimen and supporting fixture rings.

The benefits of using Lagrangian Finite Element Analysis for this design work are its ability to track history variables (for plastic deformation) and contact treatment. Based on these findings, a new configuration was developed, which consists of an array of concentric rings that support the specimen. During shock formation in the specimen, these rings progressively transfer the loading in the impact direction and radially away from the specimen, acting as momentum traps and preventing unwanted release waves from affecting the strain state experienced by the specimen.

Comparing distance time diagrams between original and proposed configurations, a design sensitivity analysis was performed, where the new geometry resulted in a decrease of both the residual velocity $(-38 \%)$ and radial displacement $(-27 \%)$ of the target when compared to the original setup.

\section{Introduction}

The plate impact experiment [1-3] is a fundamental tool in the experimental investigation of material behaviour at high strain rates. In addition to providing Hugoniot data it is increasingly used, combined with soft recovery techniques, to 
investigate the response of materials to shock loading [4-7]. Proper understanding of the recovered sample requires knowledge of the shock history experienced by the sample. Ideally the sample is exposed to a single shock compression, followed by a single release, while remaining in a state of uniaxial strain. In practice reflected waves generated at the free surfaces of the specimen and flyer plate interact and influence the strain history of the material, shown schematically in Figure 1 making it impossible to separate from the effects of the desired single shock release [8]. This lead to the development of the concept of the momentum trap [9], which in its simplest form is the addition of an additional block of material, impedance matched to the sample, that allows the transmission of the initial compressive wave from the sample without reflection but separates from the sample before undesired release waves reach the contact interface.
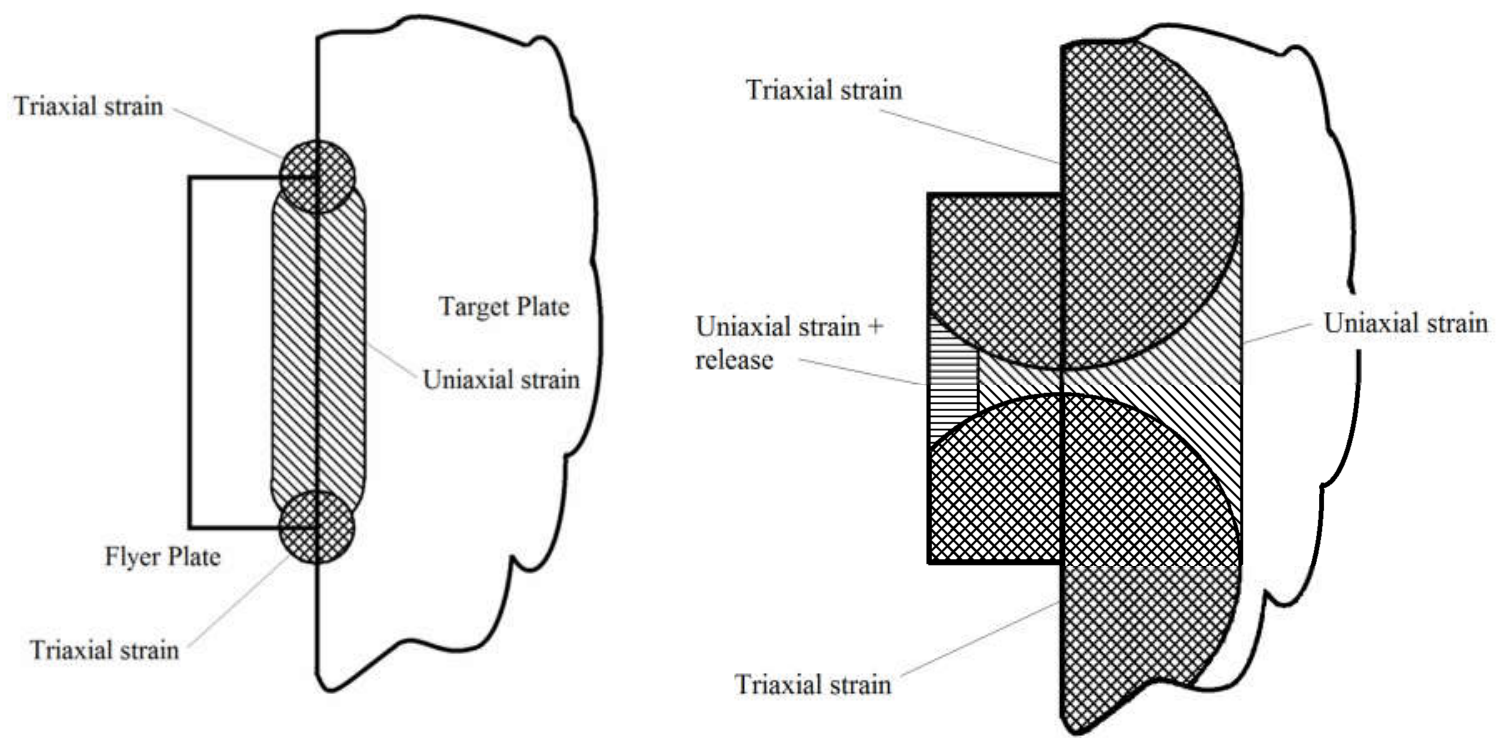

Figure 1 - Impact of a finite flyer plate onto a semi-infinite target, a few moments after impact (left) and at a later time (right)

Due to the complexity of understanding what is going on during a shock recovery test, several researchers have adopted the use of numerical analysis. An early 
investigation by Stevens and Jones [10] analysed the effects of radial release phenomena and the beneficial influence of incorporating guard rings using a twodimensional Lagrangian finite difference code. Rabie and Vorthman [8] who performed three-dimensional computer modelling in order to prove that the midsection of star shaped samples were protected from the effects of the radial release and were in a state of uniaxial strain. More recently studies by Stojkovic [15], Bourne and Gray [12] and d'Almeida et al. [6] have applied hydrocode analysis to the design of a shock-recovery target fixture.

This paper presents an analysis methodology for shock recovery fixtures based on the use of Lagrangian finite element analysis. The use of Lagrangian methods allows for natural treatment of material interfaces, including contact interfaces. The accurate treatment of the interaction of the separate components of the target fixture is critical to the accuracy of the numerical model. Additionally Lagrangian methods naturally track material history parameters, an advantage when more complex constitutive models are used. This analysis methodology is described in section 3 and is then applied to the optimisation of an improved fixture design in section 4 .

\section{Baseline experimental layout}

An initial configuration was investigated (Figure 2), which consists of nine different parts, whereby the specimen / target is surrounded by inner and outer rings, together with four spall plates and a cover plate. 


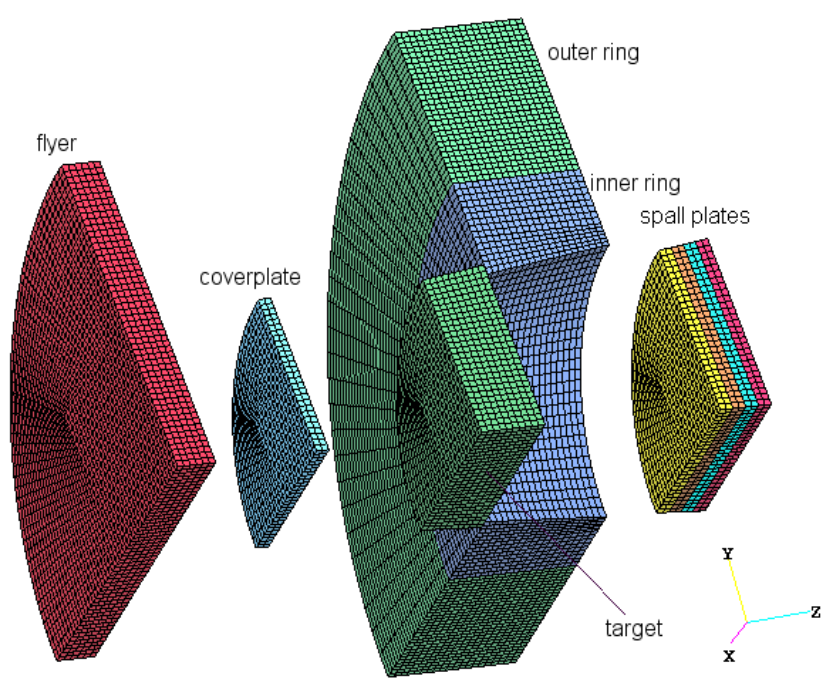

Figure 2 - Finite element discretisation of baseline configuration - low resolution model shown for clarity

With this configuration, a flyer plate whose diameter is large compared to its thickness allows planar, high amplitude waves of uniaxial strain to be generated in the central portion of the specimen, before the arrival of any release waves from the edges of the specimen. This is made possible by allowing the specimen to separate from the fixture plates and surrounding materials through the incorporation of lateral release, resulting in the trapping of tensile release waves in the surrounding components, which cannot re-enter the specimen.

The separation between parts plays a critical role, since it directly influences the propagation of a shock wave across an interface. Tolerances have to be tight in order to provide shock continuity and to prevent any reflections occurring at the interfaces between parts. Bourne and Gray [11] state their assemblies are manufactured to machining tolerances of $25.4 \mu \mathrm{m}$. In this study, the effect of machining tolerances was taken into account in the numerical analysis by introducing a $10.0 \mu \mathrm{m}$ gap between all components which is considered as an average value. 
A momentum trap behind the target (which consists of four spall plates in Figure 2) has two purposes. Firstly, it prevents any unwanted reloading, except for the initial compressive and tensile wave reflected from the backside of the flyer plate, and secondly, is used to trap part of the momentum in order to decrease the residual velocity of the specimen.

The function of the inner and outer ring (lateral momentum trap) is to constrain the target in the radial direction and reduce lateral loading of the sample. These rings have to be sufficiently sized to provide enough time for the release wave coming from the back of the flyer plate to unload the specimen and allow its separation from the fixture, before any radial release waves arrive. In addition, these rings also serve to absorb part of the impact energy.

The flyer plate for the given test set-up has a diameter of $25 \mathrm{~mm}$ and a thickness of $3 \mathrm{~mm}$ and an initial velocity of $300 \mathrm{~ms}^{-1}$. The material for all parts used in this experiment was a H04 tempered Oxygen-Free, High Conductivity Copper (OFHC).

\section{Analysis Led design using a Lagrangian Hydrocode}

This research is related to a study presented by Bourne et al, who applied an Eulerian approach to assess a similar baseline configuration [11]. Knowing the limitations of an Eulerian approach in modelling shock response, in terms of difficulties in handling contact interfaces and issues related to material tracking, whereby multi-materials are assigned to single cells, a purely Lagrangian approach was adopted in this research, as it represents a more robust approach to capturing the underlying physics.

As will be demonstrated, an explicit Lagrangian finite element code has been able to provide further insight into the problem and through the use of distance-time plots has led to significant design improvements in this shock recovery experiment. Using LSDYNA, all parts were modelled with a Johnson-Cook Elasto-Plastic Material model 
in combination with a Gruneisen Equation-of-State, as shown in Table 1, using the cm-g- $\mu$ s system of units.

Table 1: Material properties for OFHC Copper obtained from [4],[5],[6]

\begin{tabular}{|l|l|}
\hline Density & $8.94 \mathrm{~g} / \mathrm{cm}^{3}$ \\
\hline Modulus of Elasticity & $115 \mathrm{Gpa}$ \\
\hline Poisson's Ratio & 0.31 \\
\hline Shear modulus, G (GPa) & 44 \\
\hline Yield stress constant, A (MPa) & 90 \\
\hline Strain hardening coefficient, B (MPa) & 292 \\
\hline Strain hardening exponent, $\mathrm{n}$ & 0.31 \\
\hline Strain rate dependence coefficient, C & 0.025 \\
\hline Temperature dependence exponent, $\mathrm{m}$ & 1.09 \\
\hline Melt temperature, $\mathrm{T}_{\mathrm{m}}(\mathrm{K})$ & 700 \\
\hline Room temperature, $\mathrm{T}_{\mathrm{r}}(\mathrm{K})$ & 300 \\
\hline Reference strain rate, $\varepsilon_{0}(1 / \mathrm{s})$ & 1 \\
\hline Specific heat, c & 0.477 \\
\hline Velocity curve intercept, C & 0.394 \\
\hline First slope coefficient, $\mathrm{S}_{1}$ & 1.498 \\
\hline Second slope coefficient, $\mathrm{S}_{2}$ & 0.0 \\
\hline Third slope coefficient, $\mathrm{S}_{3}$ & 0.0 \\
\hline Gruneisen coefficient, $\gamma_{0}$ & 2.02 \\
\hline Firs order volume correction coefficient, a & 0.47 \\
\hline Initial internal energy, $\mathrm{E}_{0}$ & 0.0 \\
\hline Initial relative volume, $\mathrm{V}_{0}$ & 1.0 \\
\hline
\end{tabular}

The first task in modelling the experimental set-up was to define the parameters for contact, as well as undertaking a mesh sensitivity study for a simple model consisting of a flyer-plate and specimen. A summary of the key findings include:

- Axisymmetric boundary conditions were defined to reduce model size 
- A hexahedral element mesh was created for all parts. Particular attention was paid to obtaining a uniform mesh with element aspect ratio close to one. Some difficulties were encountered in matching the mesh density in the radial direction, especially for outer rings $(8,9)$ due to the different geometry (ring versus disc). Bulk viscosity was used with default parameters of 1.5 and 0.06 for quadratic and linear coefficients respectively.

- A sliding contact algorithm with friction was defined between all parts, where a user defined penalty stiffness of 0.7 was used to ensure representative contact due to the high impact velocity involved. A higher value was required in order to reduce noise in the pressure-time histories. In order to model the effect of assembly machining tolerances a $10 \mu \mathrm{m}$ gap was introduced between all components. This value was chosen as an average value based on the statement in [11] that the typical machining tolerances for these experiments are $25.4 \mu \mathrm{m}$ [11]. The authors in [11] used a $100 \mu \mathrm{m}$ gap in their numerical model using an Eulerian code.

- Figure 3 shows the model mesh used in the analyses, this mesh has 626640 elements. This results in 4 elements through the thickness of the spall plates. This mesh was chosen following a mesh sensitivity study which considered halving and doubling the element size, which resulted in meshes with 2968064 and 46376 elements. The difference in residual velocity, and lateral strain was within $10 \%$ for the medium and high resolution meshes. 
1. Flyer

2. Cover plate

3. Target

4. Spall plate 1

5. Spall plate 2

6. Spall plate 3

7. Spall plate 4

8. Inner ring

9. Outer ring

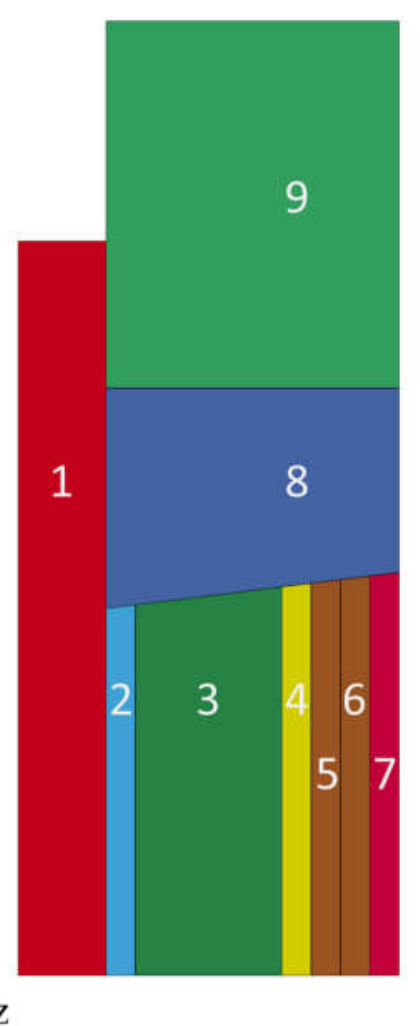

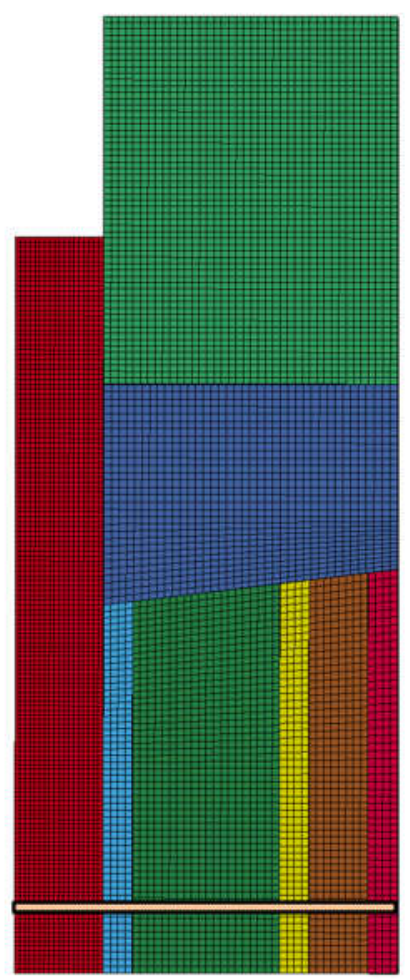

Figure 3 - Finite element representation of baseline configuration, where the set of elements highlighted represent the locations used to construct distance-time diagrams.

The distance-time diagram for the baseline configuration can be found in figure 4 , where the specimen is located between 0.1 and $0.6 \mathrm{~cm}$. It is clear from this figure that there are a number of significant flaws with the baseline configuration:

- High degree of noise / oscillation occurs at the initial impact point (A) between flyer and cover plate, and between cover plate and target plate.

- Spall plates are not working as intended due to their low thickness. There are two important observations to be made, the first is the presence of multiple short duration pulses propagating between the spall plates (B) and secondly, the superposition of the release waves originating from the rear of the flyer and the rear of the last spall plate occurs over a number of spall plates. This 
allows the release wave from the last spall plate to transmit back into the specimen before the spall plates separate. A consequence of this is the noise in the specimen at location (C).

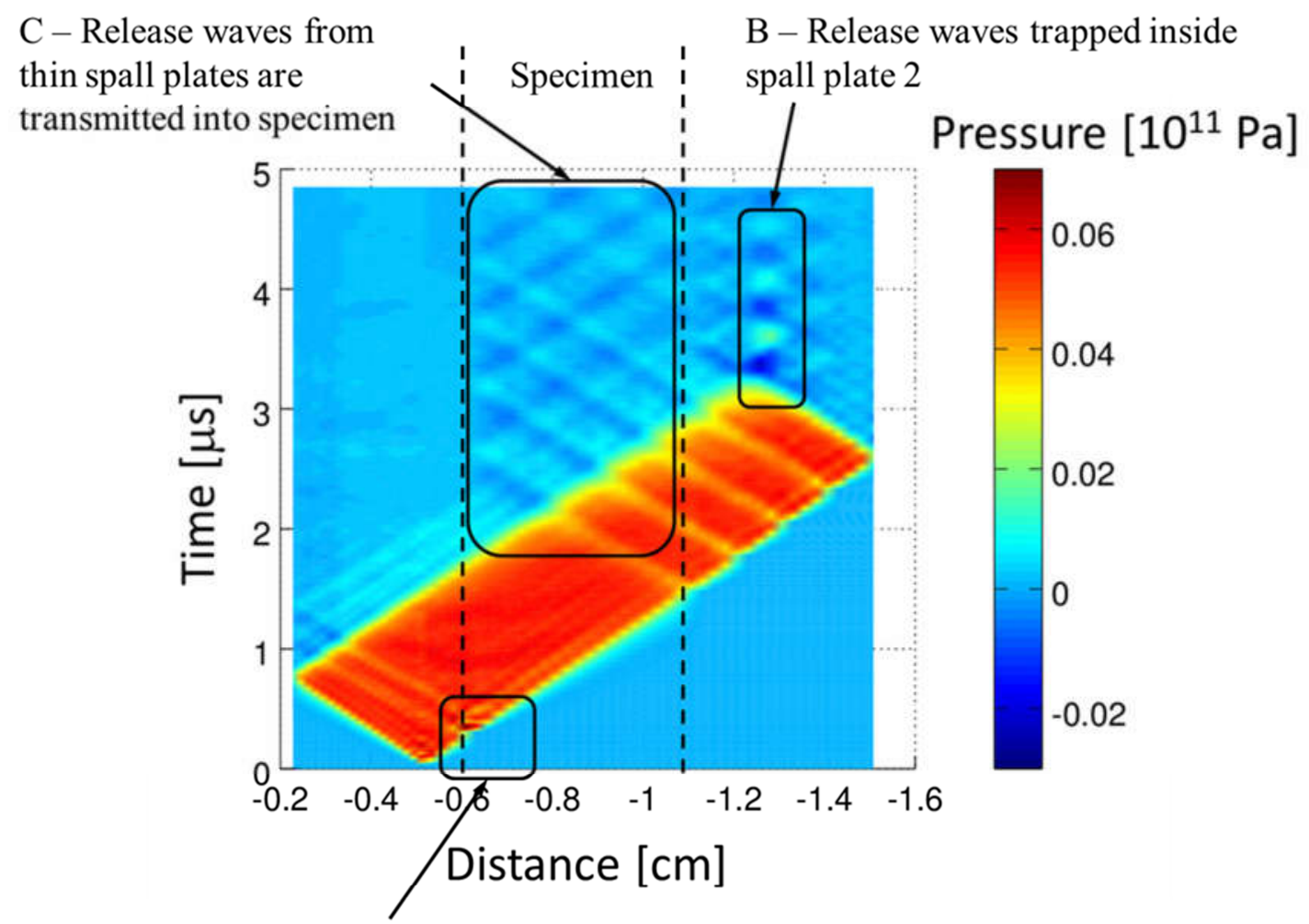

A - Contact noise at flyer-cover plate and cover plate-target interface

Figure 4 - Distance-time diagram for the baseline configuration highlighting a weakness of the design as the release waves are not fully trapped within the spall plates

In order to determine the level of influence of the radial release waves on the specimen, time histories of the lateral motion were investigated for the set of nodes that lie along the mid plane of the specimen, as shown in Figure 5. 


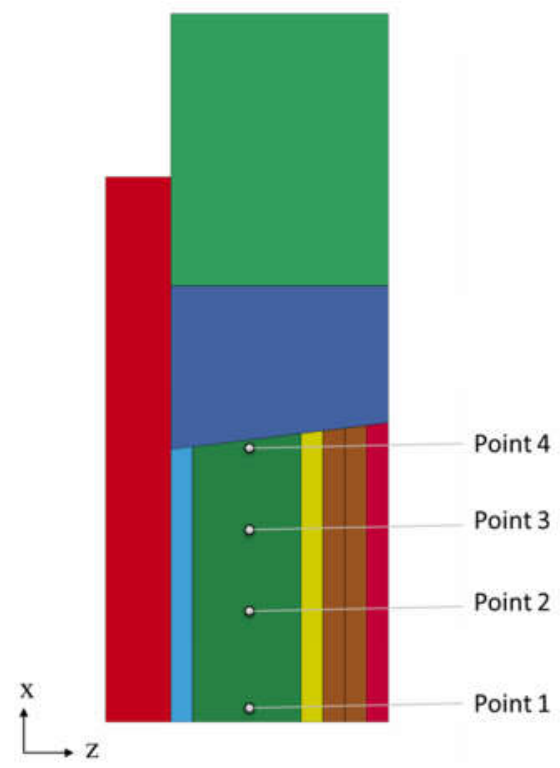

Figure 5 - Locations of the four points used to extract the time histories of lateral motion along the mid plane of the specimen for the baseline configuration

Figure 6 provides the results up to $120 \mu$ s and illustrates that lateral motion does occur in the specimen, with a maximum positive displacement of $4 \mu \mathrm{m}$. At about $55 \mu \mathrm{s}$ after initial impact the lateral displacement is affected by the contact between the specimen and inner ring, as illustrated through pressure and velocity plots in figures 7 through 9 (the impact direction is along the $\mathrm{Z}$-axis). The radial displacements become negative with values between -5 to $-15 \mu \mathrm{m}$.

The contact arising between specimen and inner ring is a result of the inner ring having a higher velocity relative to the specimen. This impingement results in a pressure concentration developing in the specimen (Figure 7), leading to additional departure from uniaxial strain state of deformation. 


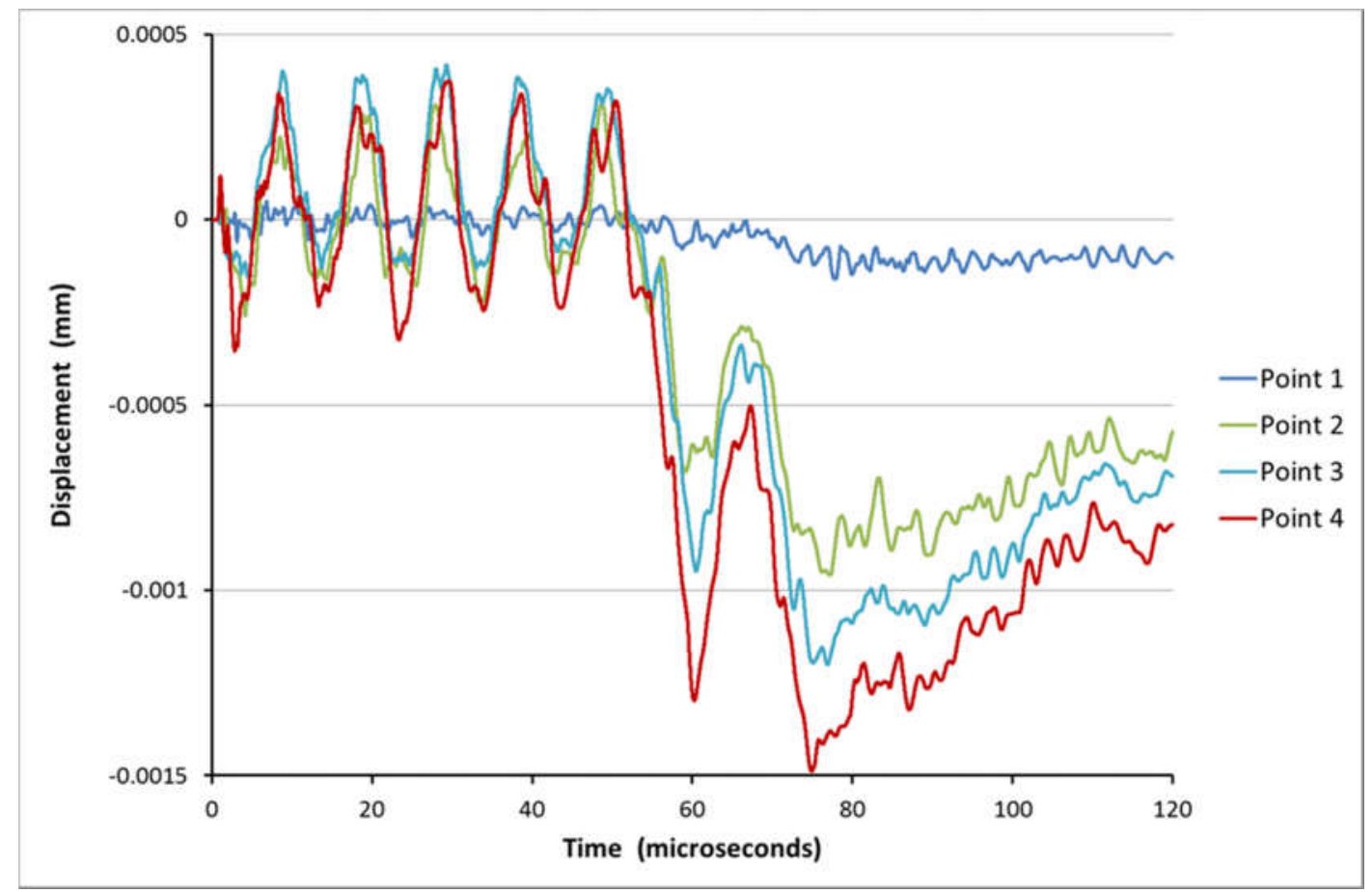

Figure 6 - Lateral displacements of nodes lying along the mid-plane of the specimen for the baseline configuration

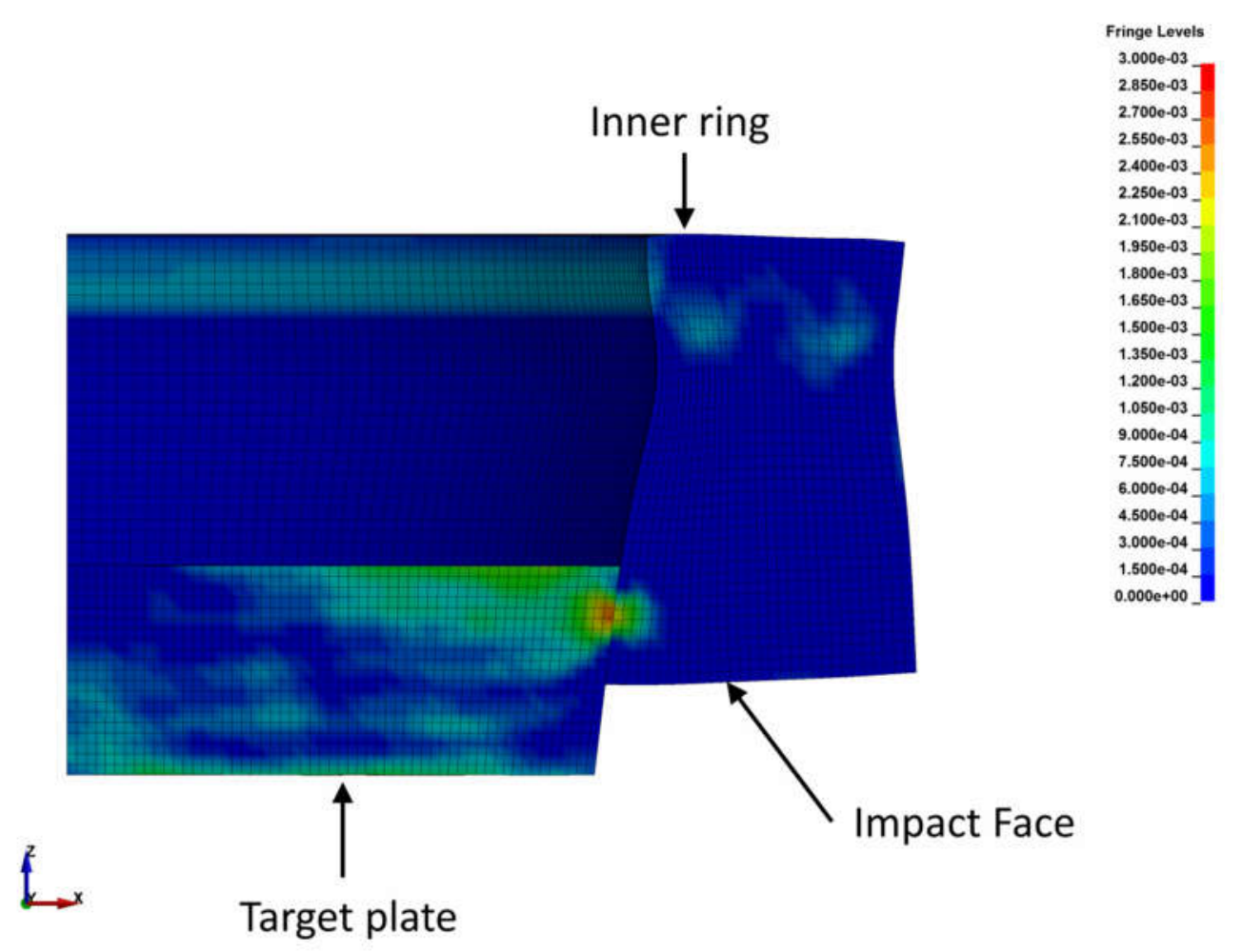

Figure 7 - Distribution of pressure $\left[10^{11} \mathrm{~Pa}\right]$ for the target and inner ring at the moment of the contact (response time $55.8 \mu \mathrm{s}$ ) 


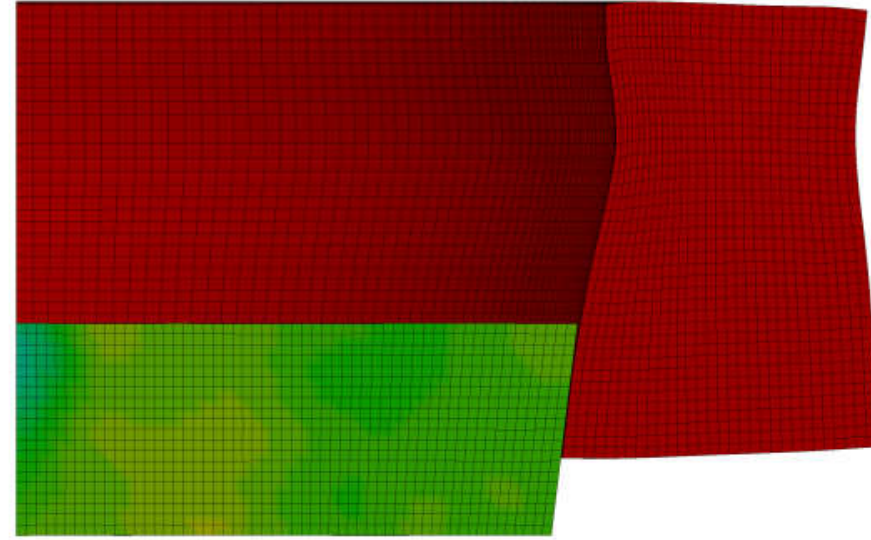

$1.950 \mathrm{e}-03$

$1.800 \mathrm{e}-03$

$1.650 \mathrm{e}-03$

13500

$1.200 \mathrm{e}-03$

$1.050 \mathrm{e}-03$

$9.000 \mathrm{e}-04$

$7.5000-04$

$6.0000-04$

4.500e-04

3.000e-04

$1.500 \mathrm{e}-04$

$0.000 \mathrm{e}+00$

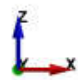

Figure 8 - Velocity profile $[\mathrm{cm} / \mu \mathrm{s}]$ before contact between the target plate with inner ring (response time $50 \mu \mathrm{s}$ )
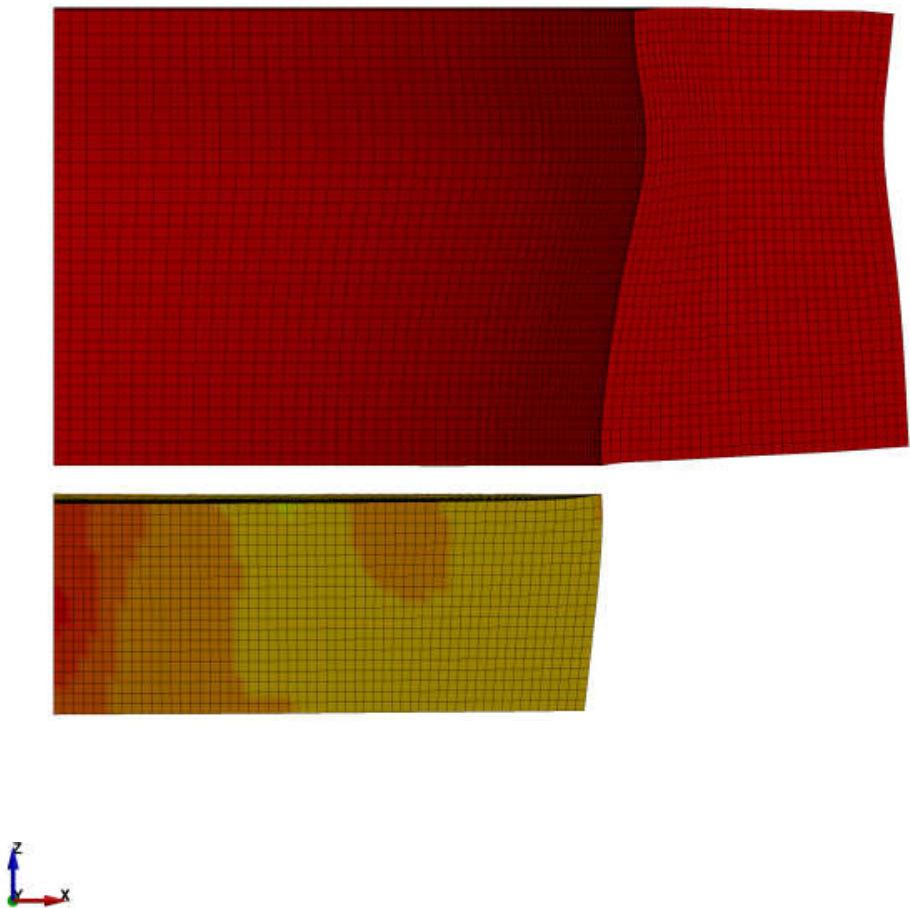

Figure 9 - Velocity profile $[\mathrm{cm} / \mu \mathrm{s}]$ when target and inner ring contact (response time $120 \mu \mathrm{s})$ 
The observed lateral motion of material in the specimen is not desirable, since it violates the uniaxial strain state. At the same time this indicates the scope for further improvement of the baseline configuration. The strain in the $\mathrm{z}$ (impact) and $\mathrm{x}$ (lateral) directions for the specimen at different response times is shown in Figures 10 and 11 respectively. Taking the impact direction first, states (a) through (c) in figure 10, represent initial compression and the evolution to a fully compressed state, before unloading, states (d) to (e). State (f) shows the distribution of strain in the $\mathrm{Z}$ direction in the sample after its secondary contact with the momentum trap ring. This impact introduces the non-uniformity in the strain field. Figure 11, illustrates influence of the radial release on the specimen. States (a) through (c) show the strain in the lateral direction during initial shock propagation (compression in the $\mathrm{x}$ direction). The tensile strain on the impact side of the specimen is nonphysical and caused by the contact algorithm. States (d) and (e) show the influence of the radial release waves on the lateral strain distribution in the specimen, during the release phase. State (f) shows the lateral strains distribution after the secondary contact between the specimen and the momentum trap ring which introduces additional non-uniformity of the lateral strain field. 


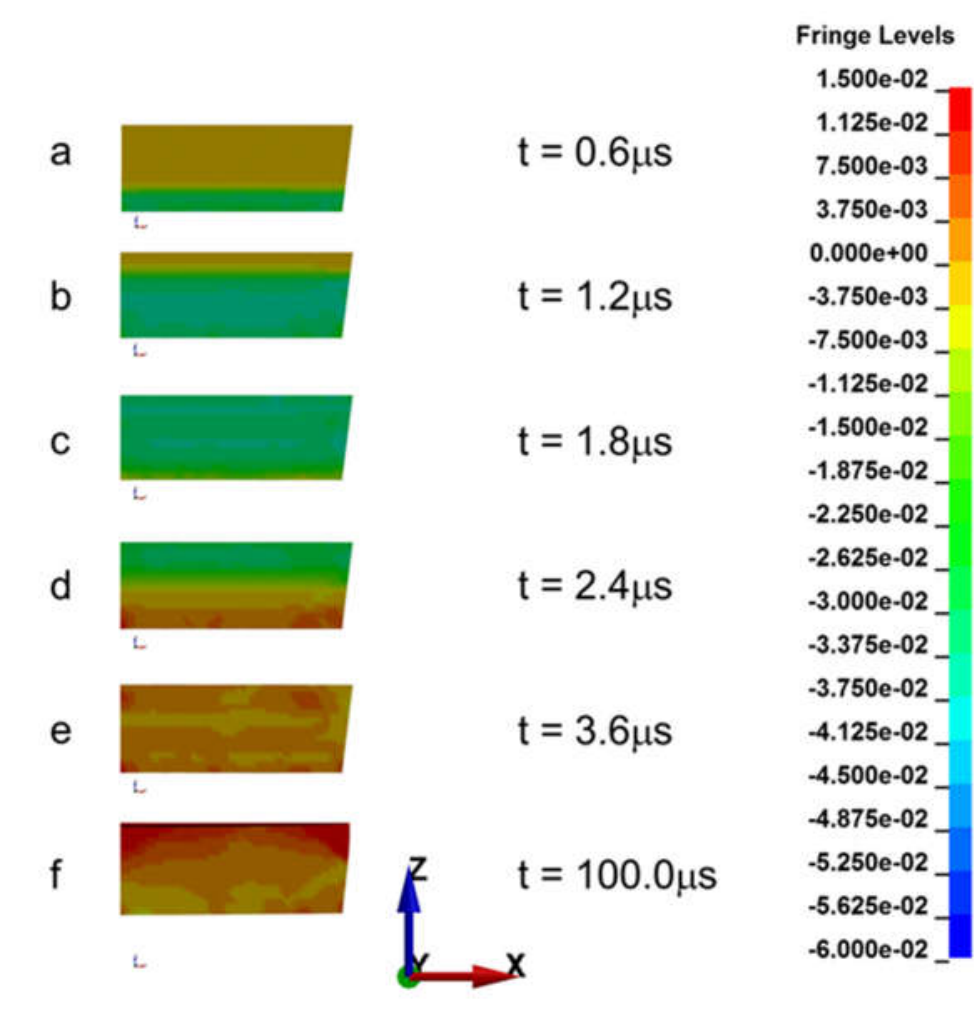

Figure 10 - The impact $(\mathrm{Z})$ direction strain in the specimen as a function of time $(\mu \mathrm{s})$

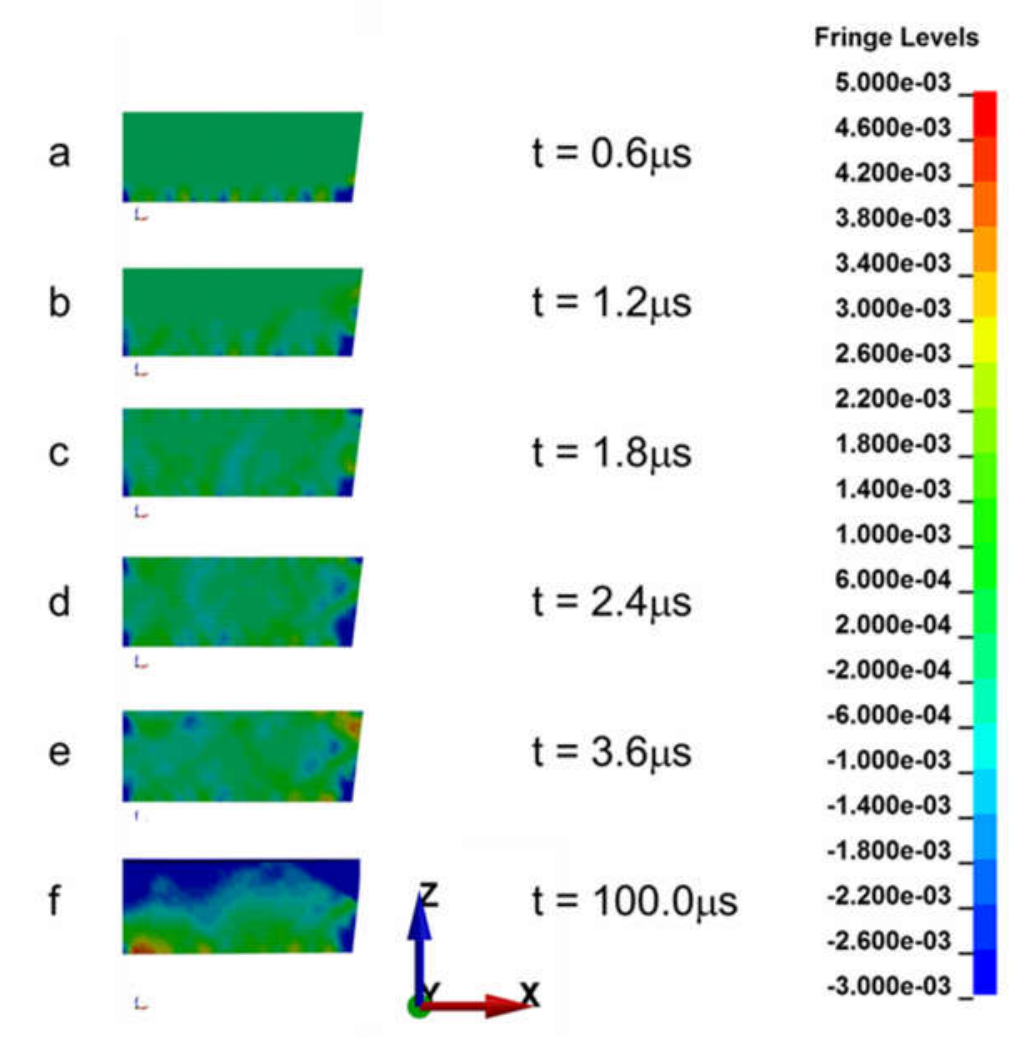

Figure 11 - The lateral (X) direction strain in the specimen as a function of time $(\mu \mathrm{s})$ 
The effects of the propagation of both longitudinal and radial release waves (Figures 10 and 11) can also be illustrated by the material loading-unloading (stress vs. strain) curve for an element in the centre of the specimen shown in Figure 12. Stages (a) through (c) represent the loading path, and states (d) and (e) represent the unloading path. Figure 12 shows that the unloading path is not parallel with the loading path, which is another indication of the influence of radial release and a departure from the uniaxial state of strain. This is clearer when looking at the difference between stress during loading and unloading for specific values of strain $(-0.005,-0.015,-0.020$, 0.025) listed in Table 2. These stress differences strains are also shown on Figure 12.

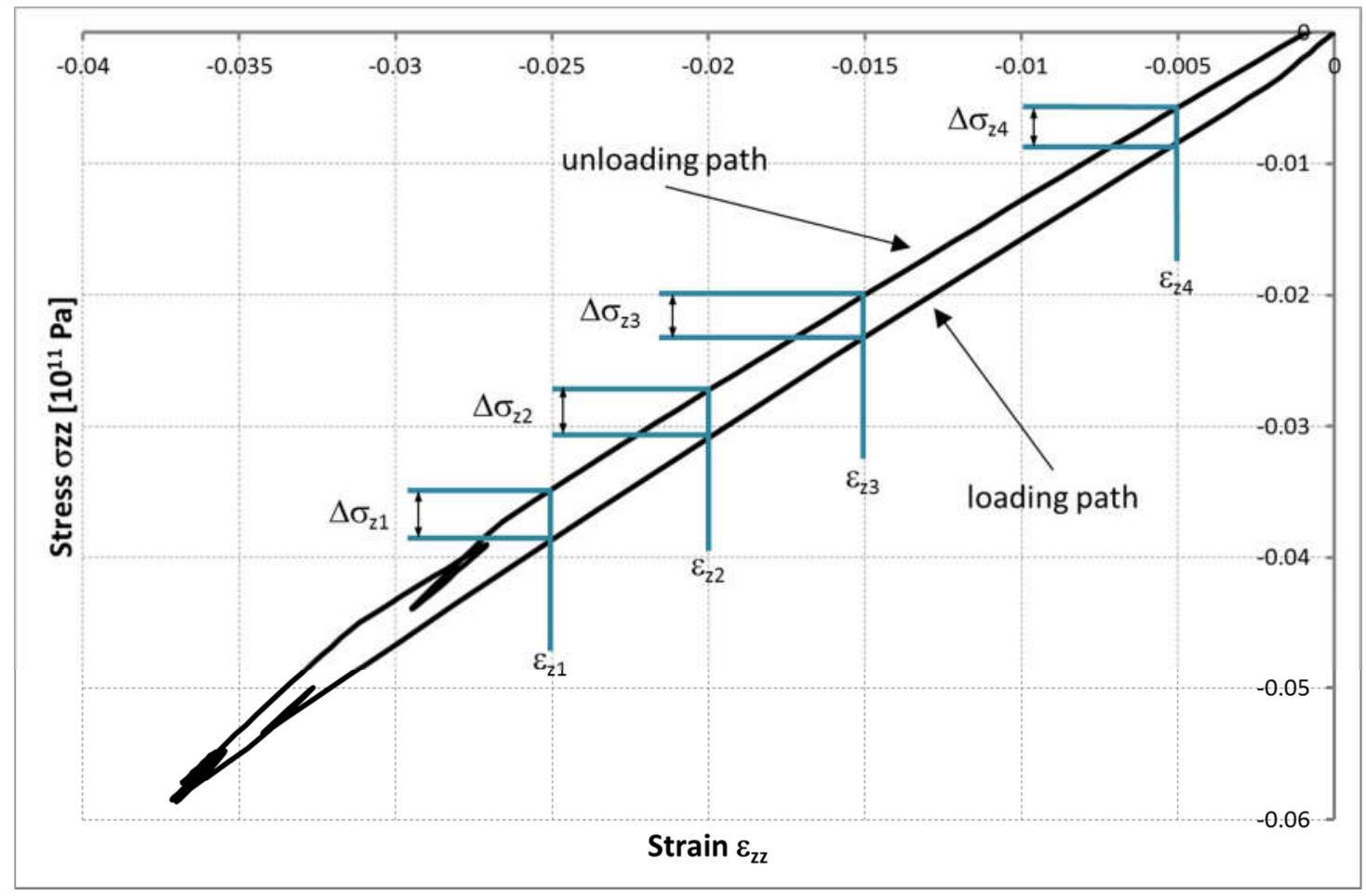

Figure $12-\mathrm{Z}$ direction stress v.s. $\mathrm{Z}$ direction strain curve, showing the loading and unloading path for a central element in the specimen 
Table 2: Difference between the loading and unloading paths

\begin{tabular}{|c|c|c|c|c|}
\hline & $\begin{array}{c}\varepsilon_{\mathrm{z} 1}=-0.025 \\
\Delta \sigma_{\mathrm{z} 1}\left(10^{11} \mathrm{~Pa}\right)\end{array}$ & $\begin{array}{c}\varepsilon_{\mathrm{z} 2}=-0.02 \\
\Delta \sigma_{\mathrm{z} 2}\left(10^{11} \mathrm{~Pa}\right)\end{array}$ & $\begin{array}{c}\varepsilon_{\mathrm{z} 3}=-0.015 \\
\Delta \sigma_{\mathrm{z} 3}\left(10^{11} \mathrm{~Pa}\right)\end{array}$ & $\begin{array}{c}\varepsilon_{\mathrm{z} 4}=-0.005 \\
\Delta \sigma_{\mathrm{z} 4}\left(10^{11} \mathrm{~Pa}\right)\end{array}$ \\
\hline$\Delta \sigma$ & 0.00386 & 0.00359 & 0.00319 & 0.00263 \\
\hline $\operatorname{Time}(\mu \mathrm{s})$ & 2.21 & 2.28 & 2.34 & 2.45 \\
\hline
\end{tabular}

Another important aspect to this experiment is specimen recovery, where it is desirable for the specimen to have a low residual velocity. Based upon the initial total energy of 590J, the velocity time histories were obtained for cover plate, specimen and first spall plate located next to the specimen. The longitudinal velocity of these parts, shown in Figure 13, clearly shows the separation between specimen and cover / spall plate. After $3.5 \mu \mathrm{s}$, the residual velocity of the specimen for the baseline configuration is $17.5 \mathrm{~ms}^{-1}$. However due to the secondary impact with the momentum trap ring the target is accelerated and reaches a final velocity of $23.6 \mathrm{~ms}^{-1}$.

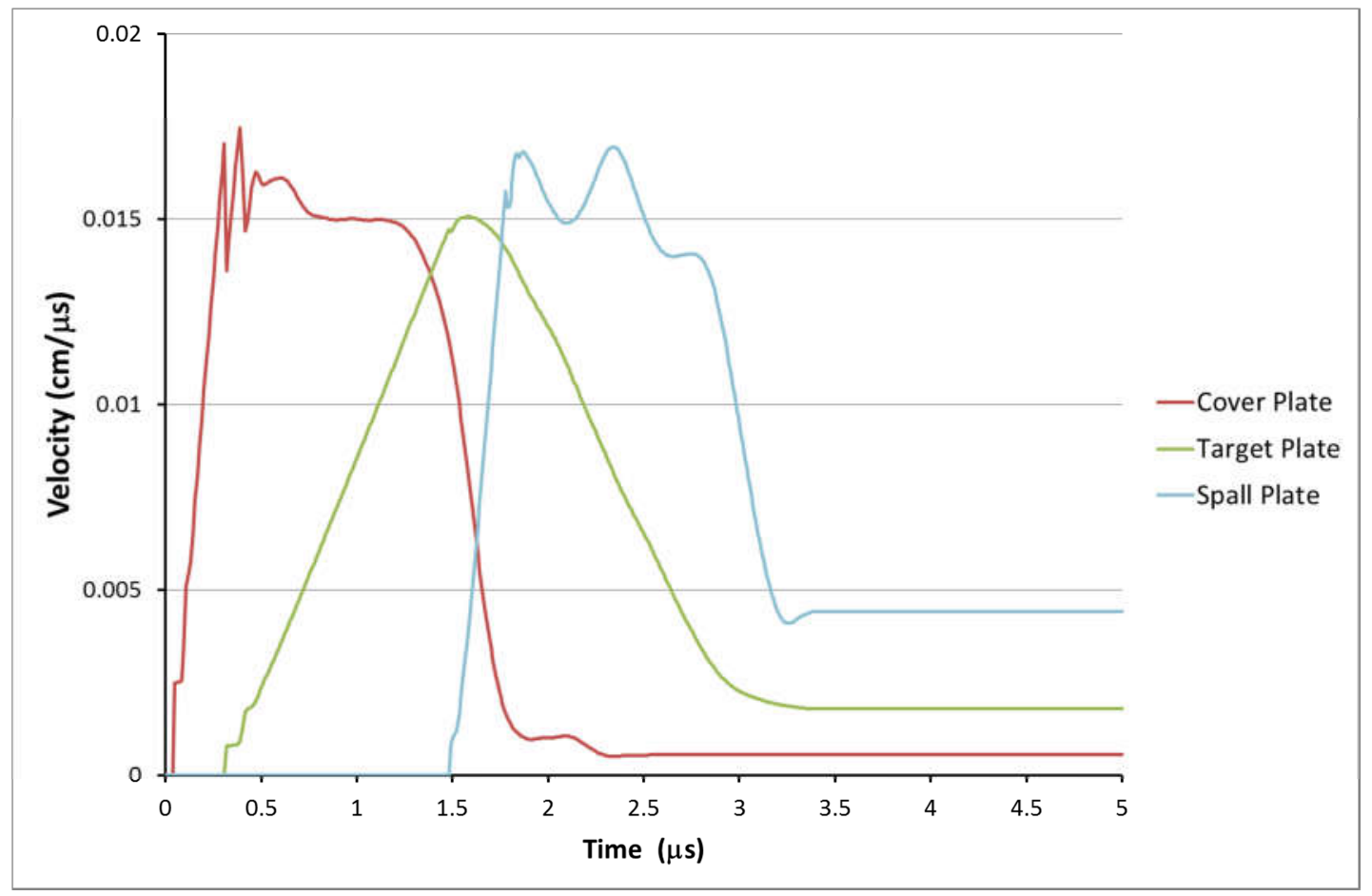

Figure 13 -Velocity-time diagram for the cover plate, specimen and spall plate for baseline configuration 


\subsection{Validation of modelling approach}

Since velocity experimental data was not available to validate these results, alternative ways of assessing whether these predicted residual velocities are realistic were sought. Experiments of similar plate impact tests which include measurements of target plate velocity using line-imaging VISAR (LIV) instrumentation were identified [12, 13, 14]. The use of LIV instrumentation provides velocity time-history data along a line on the back surface of the sample. The experimental data used for validation was published by Furnish, Gray and Bingert [12]. This data was selected because the experiment was performed on OFHC copper, the same material as in this paper. Therefore, the same material constants were used. The model consists of a $2.54 \mathrm{~mm}$ thick flyer plate, $5.08 \mathrm{~mm}$ thick target plate, three spall rings and supporting rings. Flyer plate, target plate and spall rings were all made out of OFHC copper. The spall rings are supported on a PMMA ring backed with a steel ring as shown in Figure 14.

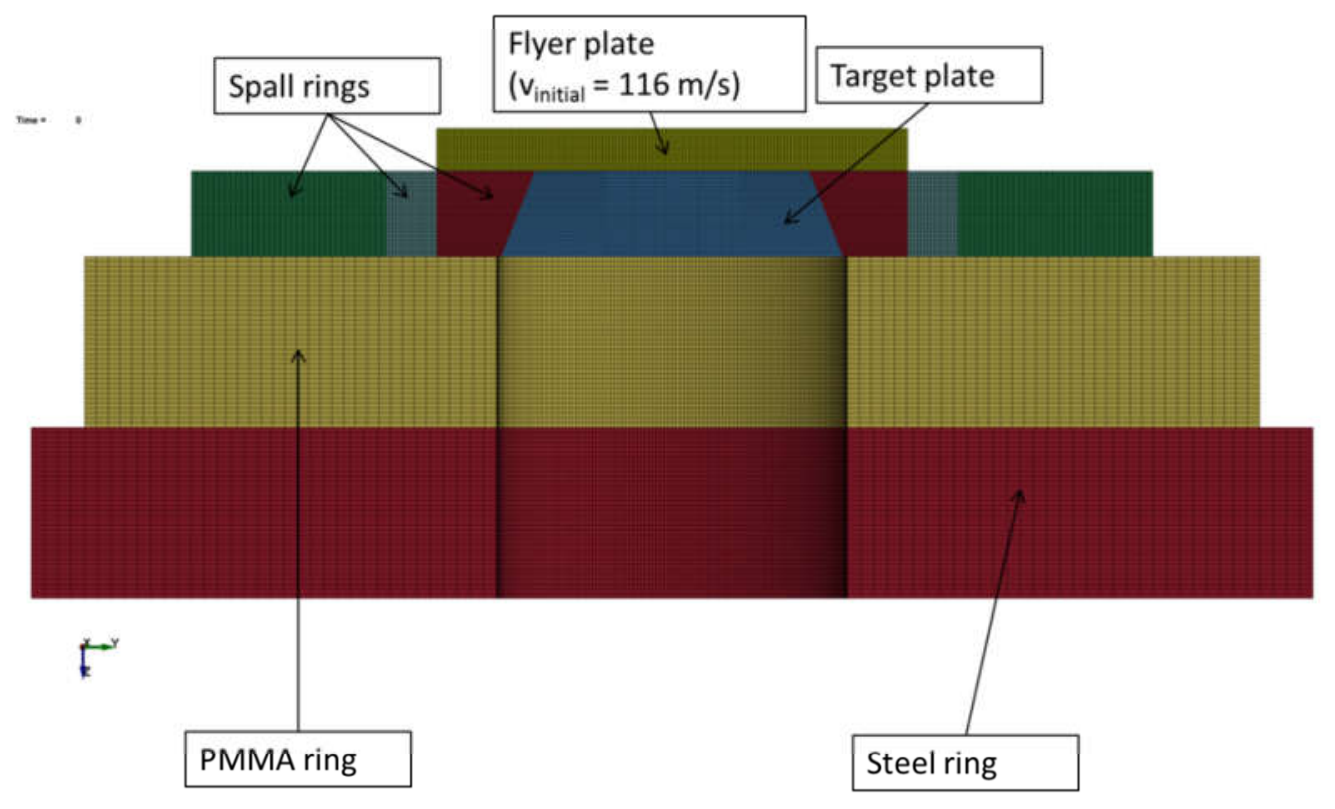

Figure 14 - Finite element model of LIV experiment 
The interaction between all parts in the model is through contact. The flyer plate impact velocity was $116 \mathrm{~m} / \mathrm{s}$, which resulted in spallation occurring in the target. To represent this, a maximum principal stress based spall model was included, where the spall strength was set to $1.5 \mathrm{GPa}$. This ensures that spall occurs when the maximum principal stress in an element exceeds this value.

In the experiment, LIV data was recorded along a $2 \mathrm{~mm}$ line on the back of target plate providing a range of velocities depending on position. This is represented by the average velocities and the lower and upper velocity bounds in a velocity versus time plot in Figure 15. This means that, for example, the lower bound velocity is the lowest velocity which occurs at any position along the measurement line at a given moment in time. Based on the data provided in [12] the velocity versus time curve for the target plate centre is also shown in Figure 15. The comparison between experimental and the simulation velocity time histories is shown in Figure 15. The simulation results consist of 7 individual velocity time histories recorded at various locations within a $2 \mathrm{~mm}$ distance from the target plate principal axis of symmetry. The numerical data was not filtered and was sampled every $0.003 \mu$ s. It can be seen that up to the point where damage and spallation starts (about response time $=2 \mu \mathrm{s}$ ), the predicted velocity pulse matches the measured velocity at the plate centre. However the variation in velocity present in the experimental data was not observed in simulation results. This may be due to the problems with the VISAR measurements in terms of point to point and time dependent intensity variations reported in [12]. The simulation recovery signal was less accurate, as expected due to the simple spall model used in this work and as such, was not the focus of this validation exercise. Overall, it can be concluded that the proposed type of model and the modelling 
approach is capable of predicting velocities in plate impact tests, and hence can be used to design shock recovery experiments and in particular optimise geometry of longitudinal and lateral momentum traps for a given specimen material.

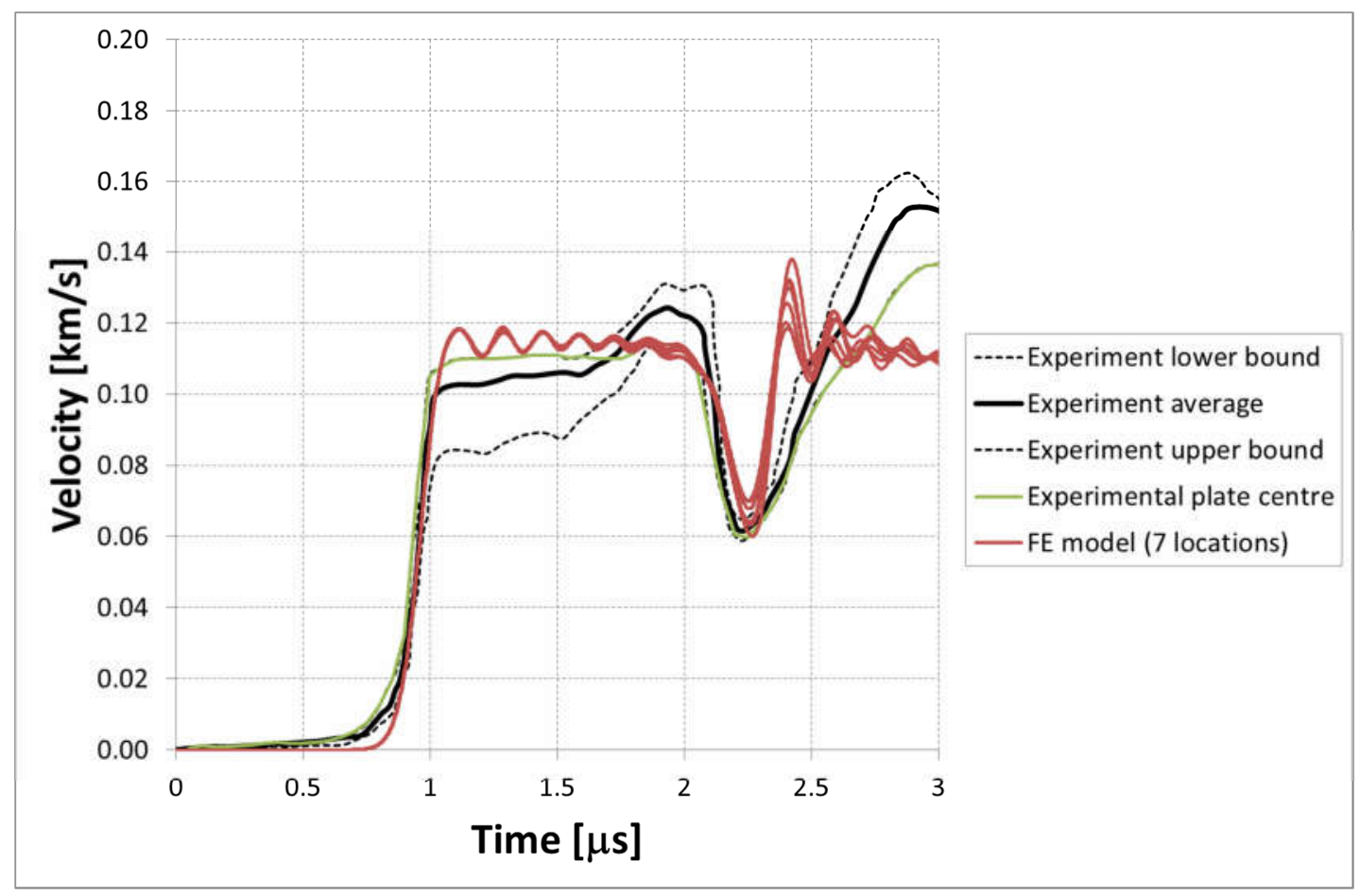

Figure 15 - Comparison of experimental [12] and numerical velocity-time results for a range of radial positions.

\subsection{Summary of Baseline Configuration Assessment}

The performance of the baseline configuration was characterised by the following:

- Undesirable contact between specimen and inner ring occurred due to the higher velocity of the inner ring. This resulted in radial deformation affecting the uniaxial strain state in specimen.

- Spall plates are too thin, as release waves are not localised in the spall plate, but have sufficient time to propagate back into the specimen before the spall plate and specimen separates. 
- High specimen residual velocity of $23.6 \mathrm{~ms}^{-1}$

These findings were the basis for the design improvements needed to overcome these shortcomings.

\section{DEVELOPMENT OF AN IMPROVED DESIGN}

Since the biggest problem with the initial design was caused by contact between the specimen and the inner ring, a solution was sought to overcome this. The first parameter investigated was theta1, the angle of the cone defined in Figure 16. Angles in range between 7-30 degrees were investigated. The results from this investigation showed that the results were still affected by unwanted contact between the specimen and inner ring.

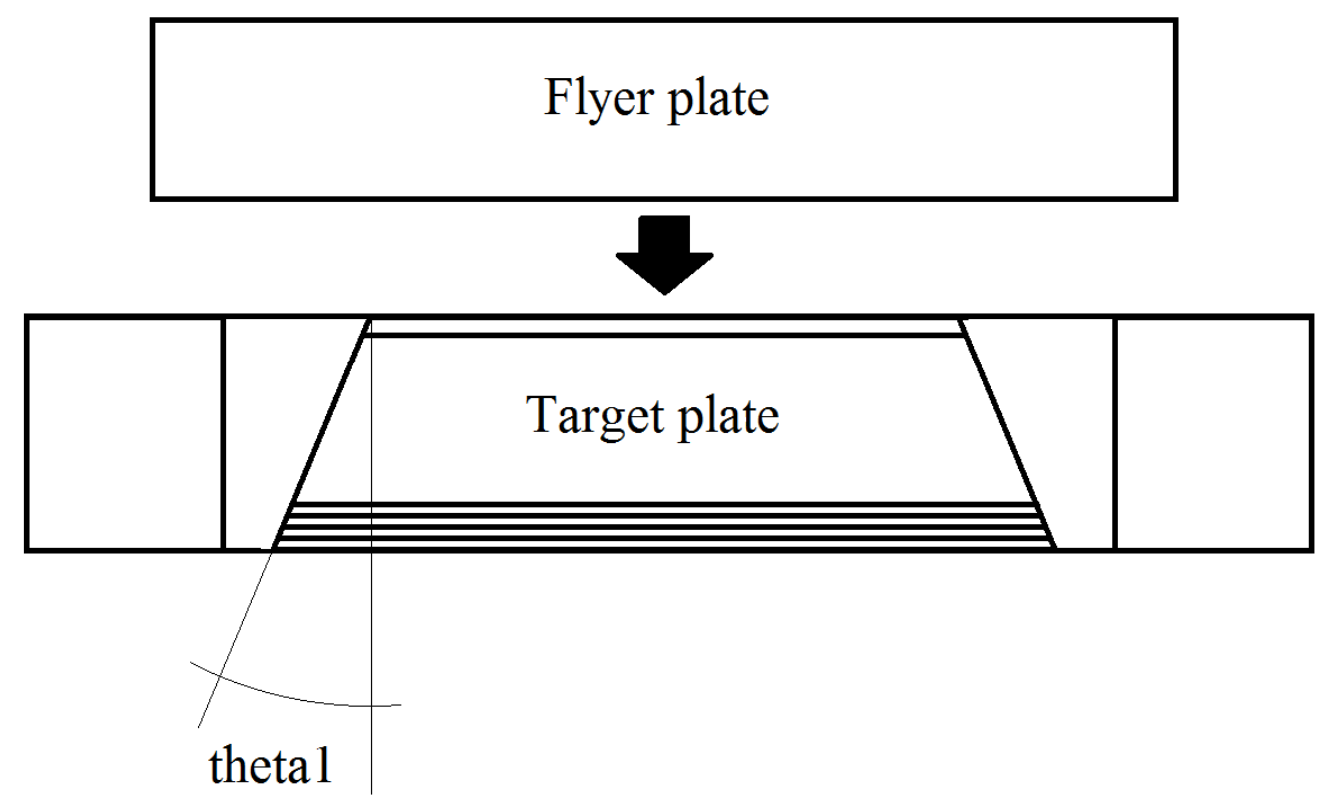

Figure 16 - First proposed solution to overcome contact arising between specimen and inner ring (this did not allow for clean separation)

As changing the thetal angle does not allow a clean separation between the specimen and the inner ring, a new geometry, shown in Figure 17, was investigated. The main 
difference from the baseline setup is that angle marked as the angle, theta2 in Figure 17, was introduced for the specimen and cover plate surfaces in contact with the inner surface of the inner ring. The idea was to employ this angle so that the inner ring does come in contact with the specimen after initial separation. Initially the angle theta2 used for the specimen and the cover plate had the same value as thetal in the baseline configuration, but it was oriented in the opposite direction.

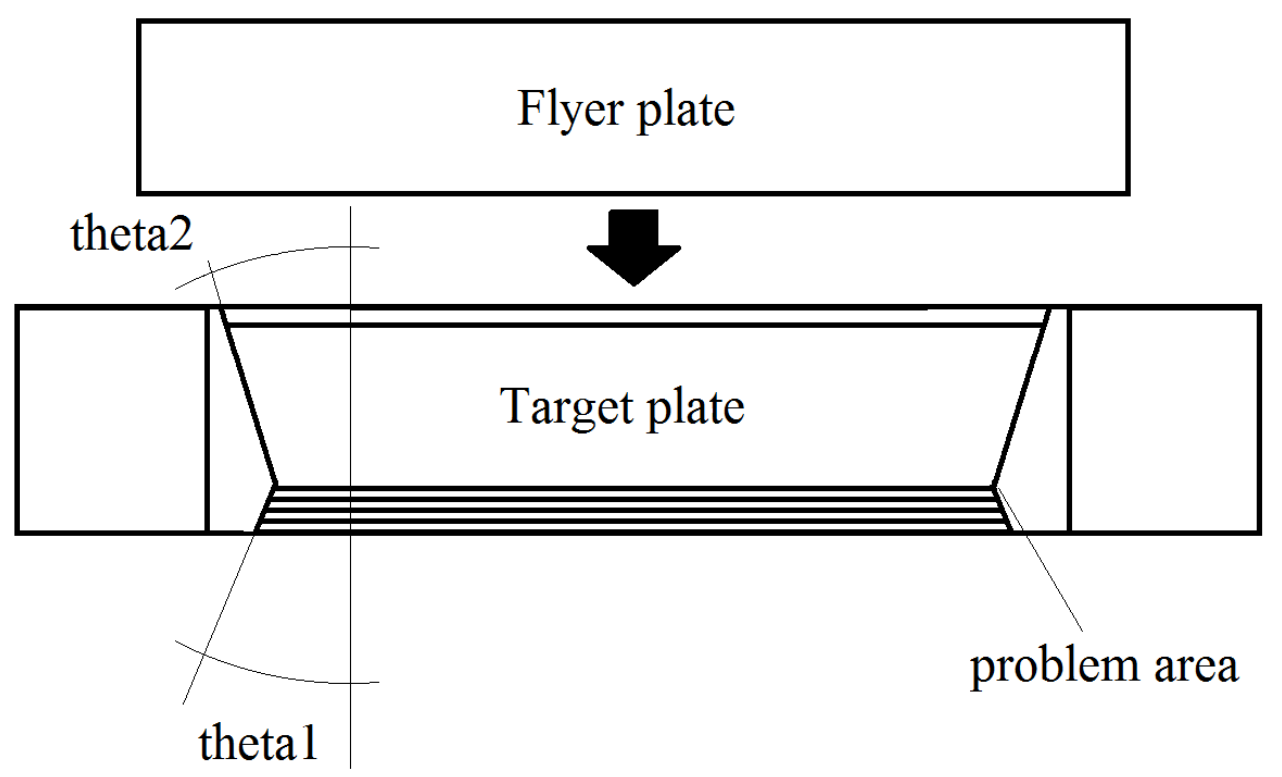

Figure 17 - Second proposed design intended to overcome contact arising between specimen and inner ring (this caused deformation of the specimen)

This configuration did successfully resolve the problem of the secondary contact, but a new problem appeared whereby the specimen was pushed through the "neck" formed at the intersection of the two opposite cones in the inner ring. The specimen therefore experiences a non-uniaxial strain. This problem was observed for the whole range of angles from 7 to 30 degrees. 
Due to this problem a further refinement of the design was required that allows both separation of the specimen and inner ring, and protection from the effects of being pushed through the "neck". The new geometry shown in Figure 18 was proposed.

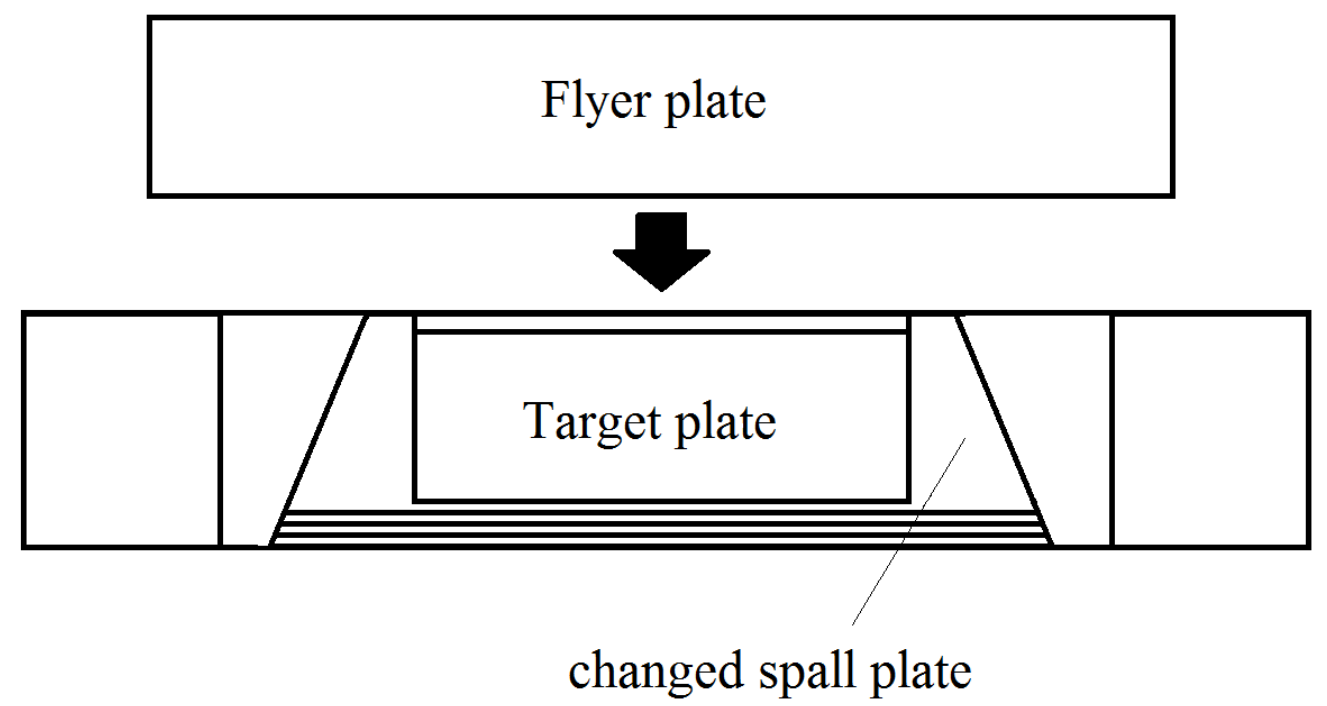

Figure 18 - Third proposed design intended to overcome contact arising between specimen and inner ring

In this design, the first spall plate was extended to encompass the specimen and protected it from the inner ring contact. This has the added benefit that it absorbs more energy due to the fact that the mass of the inner ring assembly is increased. The simulation of this geometry showed a significant advantage regarding the problem of interaction between inner ring and specimen. The inner ring has higher velocity than the target, but they cannot come into contact because of this spall plate protection. 


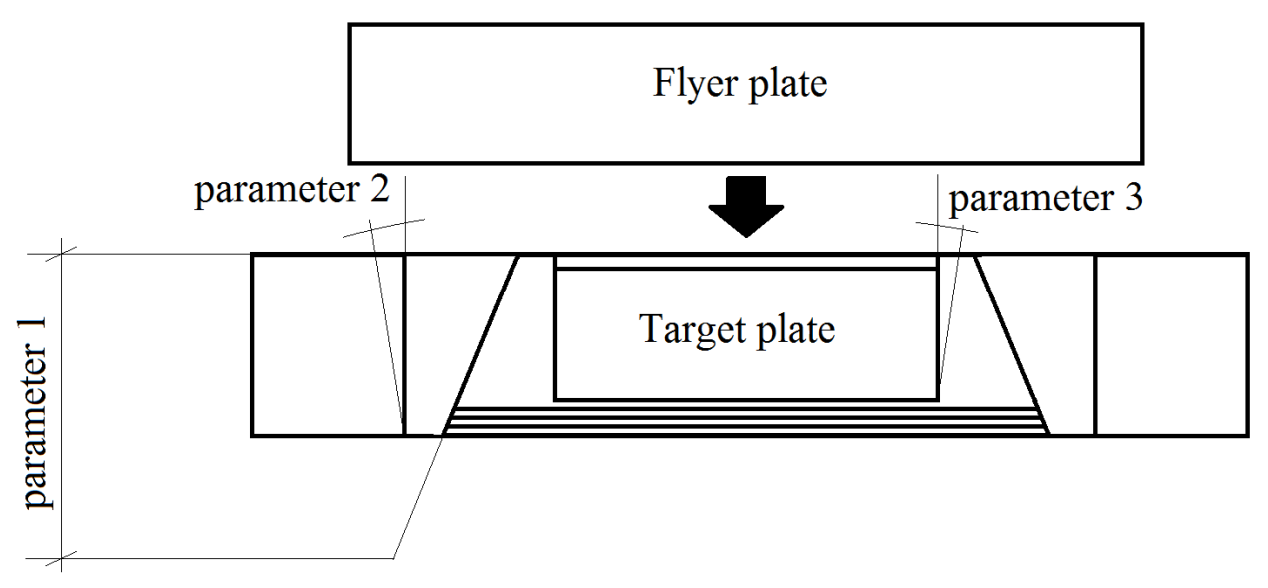

Figure 19 - Parameter to be varied in sensitivity study of third proposed design

This geometry is more effective in decreasing the radial release of the target because the material surrounding the specimen absorbs more of the initial impact energy. This results in lower velocity of the specimen and simplifies its recovery. For the parameters 1 (thickness of inner and outer rings), 2 (angle that defines outer surface of inner ring) and 3 (angle that defines surface of the specimen) shown in Figure 19, a sensitivity analysis was performed in order to optimise the proposed configuration.

The sensitivity analysis of parameter 1 representing thickness of inner and outer ring showed that this is not an efficient method of controlling both the residual velocity of the specimen and its radial release. Changing angle 2 and 3 in the manner shown in Figure 19, allows the amount of the radial release to be reduced.

Figure 20 illustrates the influence of changing angle 2. By increasing this angle, the displacement in the radial direction in the specimen material decreases. The response appears to be relatively insensitive to angles greater than 10 degrees. 


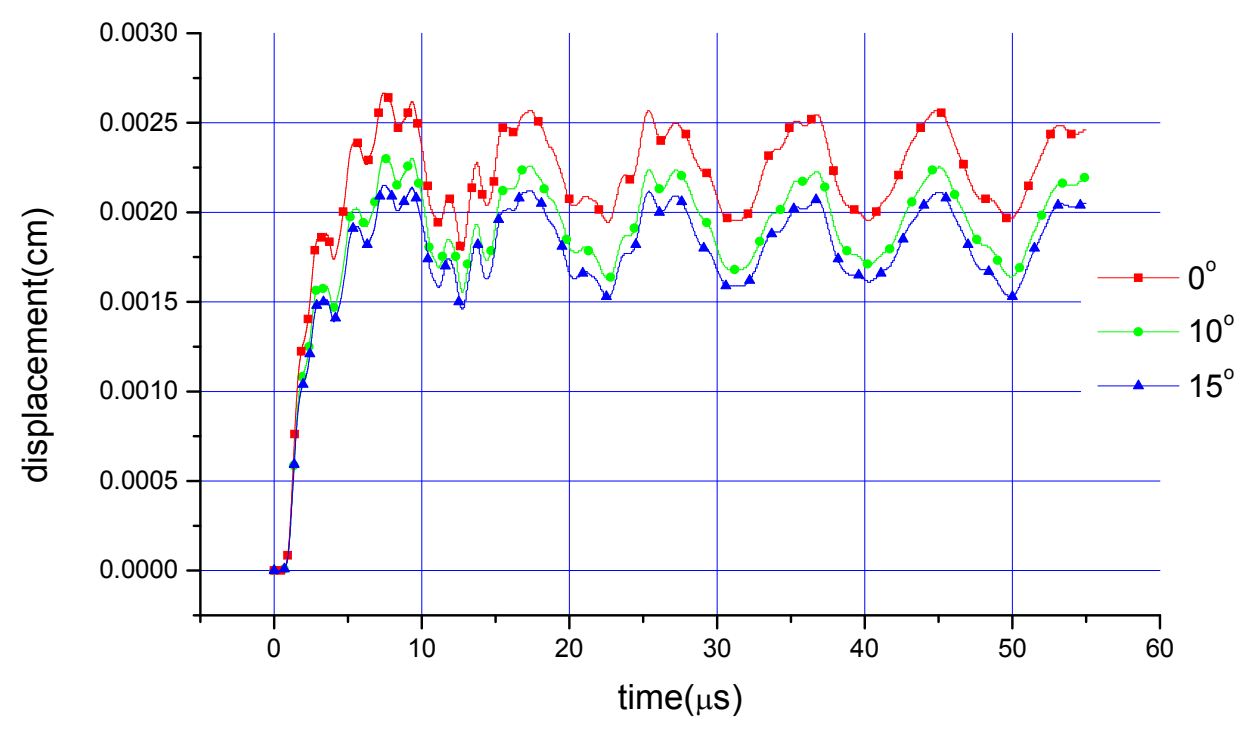

Figure 20 - Influence of change of angle 2 to the radial displacement in the specimen

The sensitivity analysis of angle 3 is presented in Figure 21, and shows a significant change in specimen behaviour in the radial direction. It can be seen that radial release decreases as the angle increases.

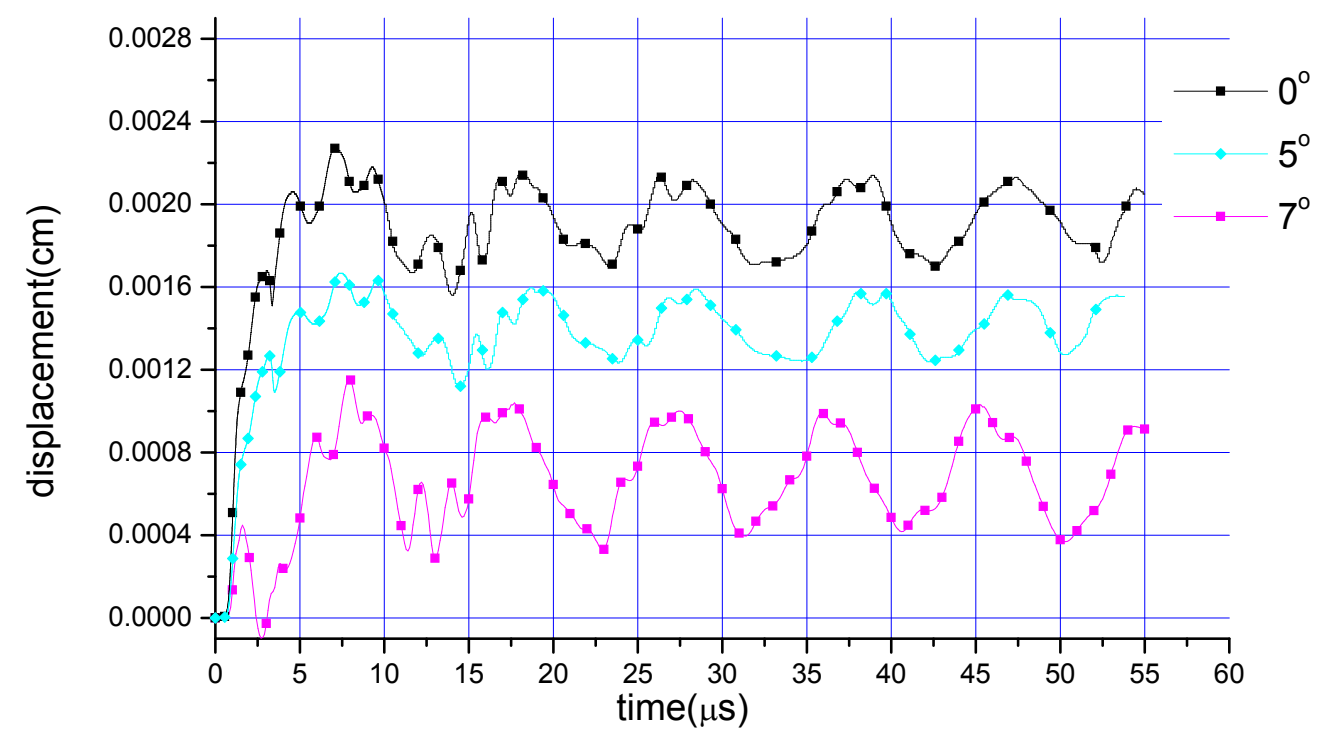

Figure 21 - Influence of change of angle 3 to the radial displacement in the specimen 
A constraint on the design is that the overall thickness of the cover plate, specimen, and spall plates should not be changed because of the location where the two release waves interact. Therefore, the thicknesses of the spall plates were adjusted such that the interaction of the two release waves occurs within fewer spall plates. The distance time diagram analyses resulted in an increase of spall plate thickness from 1 to $2 \mathrm{~mm}$. This was achieved in practice by reducing the number of spall plates, i.e. combining spall plate 1 and 2 .

All of these changes were combined into a new experimental setup. The performance of this new setup is analysed in Section 5.

\section{Numerical assessment of the optimised design}

Two main objectives were defined for this new configuration, namely:

1. Minimise the residual velocity of the specimen

2. Reduce the amount of radial release observed in the specimen

The geometry was changed according to the results from the sensitivity analysis presented in section 4 and is shown in Figure 22. The new configuration uses three spall plates (with thicknesses of 2-1-1mm respectively) instead of four spall plates of uniform thickness $(1 \mathrm{~mm})$. The flyer has a thickness of $3 \mathrm{~mm}$ and diameter of $50 \mathrm{~mm}$. The target has a thickness of $5 \mathrm{~mm}$. The cover plate a thickness of $1 \mathrm{~mm}$ and diameter $25 \mathrm{~mm}$. Spall plate 1, the inner ring and outer ring have, at the impact face location, outside diameters of respectively 28, 46 and $65 \mathrm{~mm}$. The values for parameter 2 and parameter 3 angles were $8.5^{\circ}$ and $7^{\circ}$. All simulation parameters and mesh densities were the same as in the baseline configuration model. 
1. Flyer

2. Cover plate

3. Target

4. Spall plate 1

5. Spall plate 2

6. Spall plate 3

7. Inner ring

8. Outer ring
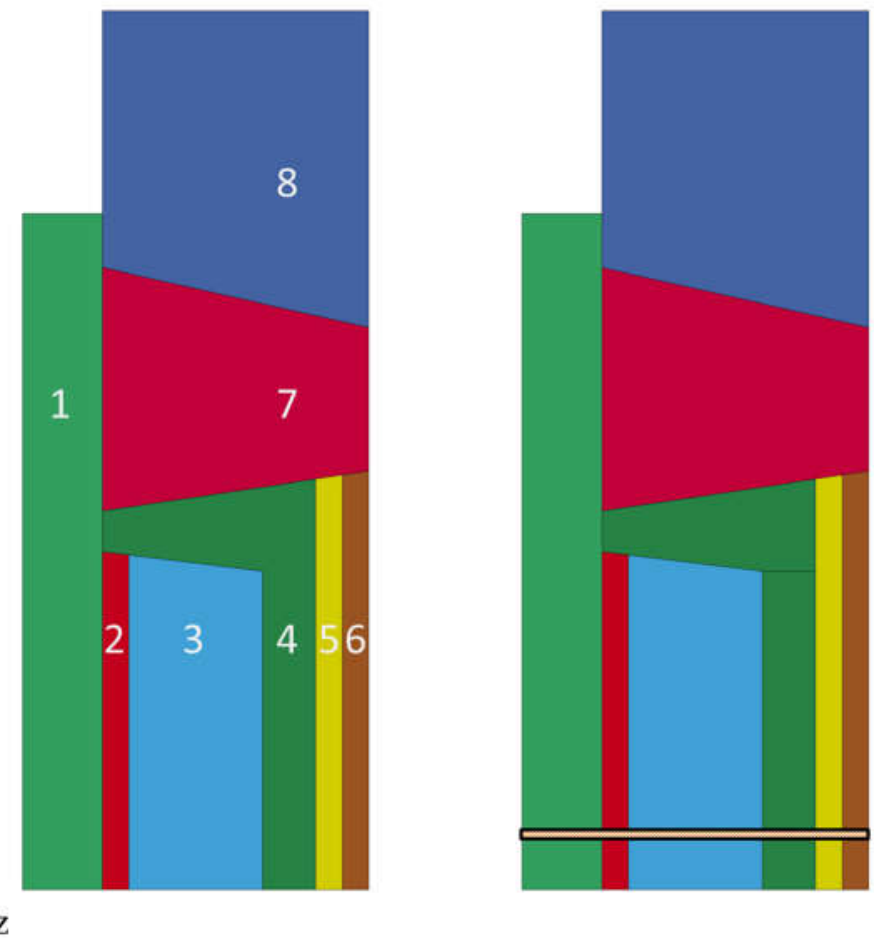

Figure 22 - Finite element representation of the optimised configuration, where spall

plate 1 is twice as thick as spall plates 5 and 6

Changing the thickness and number of spall plates now results in longer interaction of the two release waves within modified spall plate 1 (located between 0.6 and $0.8 \mathrm{~cm}$ ), which can be observed in the distance-time diagram at Point (B) in Figure 23. Furthermore, increasing the number of radial momentum traps from two to three reduced the radial release wave magnitude. The revised geometry also reduced reflections and oscillations between parts during the compression phase, such as the contact noise at point (A), together with reflections from free surfaces within the specimen at distance between -1.1 and $-0.6 \mathrm{~cm}$; both of which show a reduction when compared to Figure 4. 


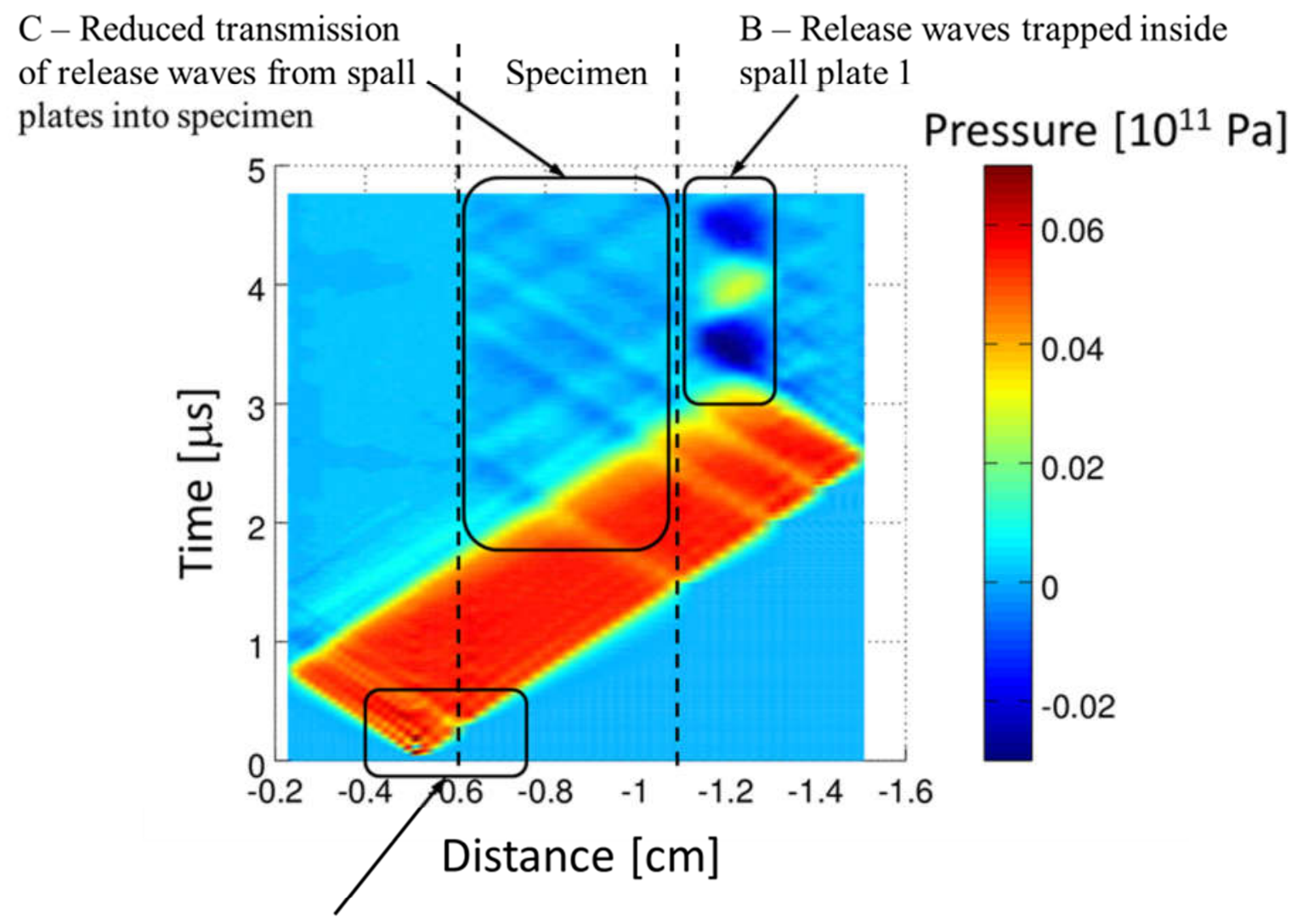

A - Reduced contact noise at cover plate-target interface

Figure 23 - Distance-time diagram of the optimised setup.

For comparison with the baseline, lateral displacement time histories were constructed for the set of nodes located along the mid plane of the specimen shown in Figure 24. Figure 25 represents a $120 \mu$ s time histories for the nodes identified in Figure 24, where it can be verified that the motion of the nodes were no longer disturbed by any additional contacts between the specimen and the inner ring. In fact, the nodes oscillate around a steady state, with the maximum displacement of $2.5 \mu \mathrm{m}$ occurring at the boundary, which represents a deformation smaller than $0.1 \%$. This is a reduction of $27 \%$ in comparison to the maximum displacement of $15.0 \mu \mathrm{m}$ observed for the baseline configuration. 


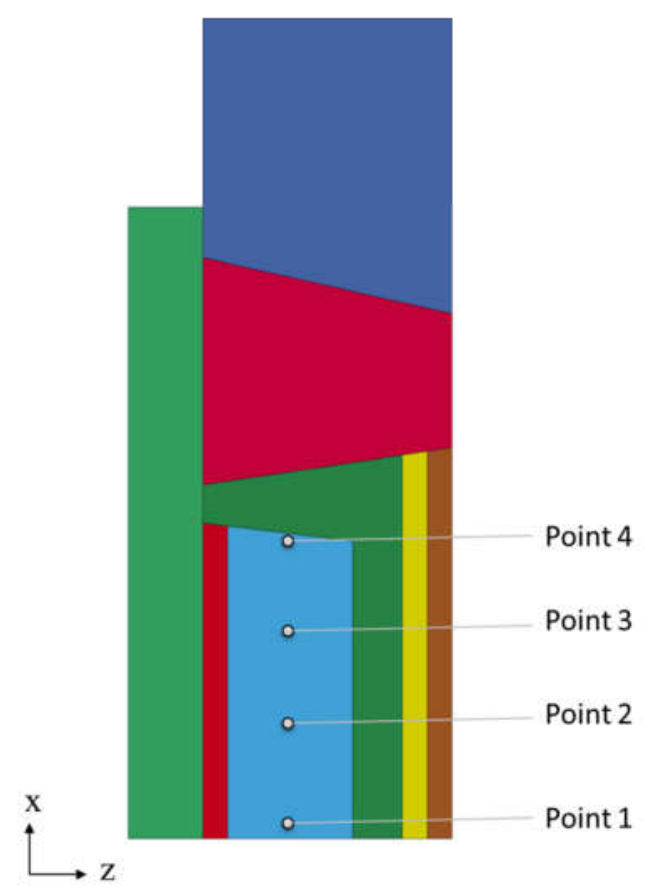

Figure 24 - Nodes used to extract the time histories of lateral motion along the mid plane of the specimen for the optimised configuration

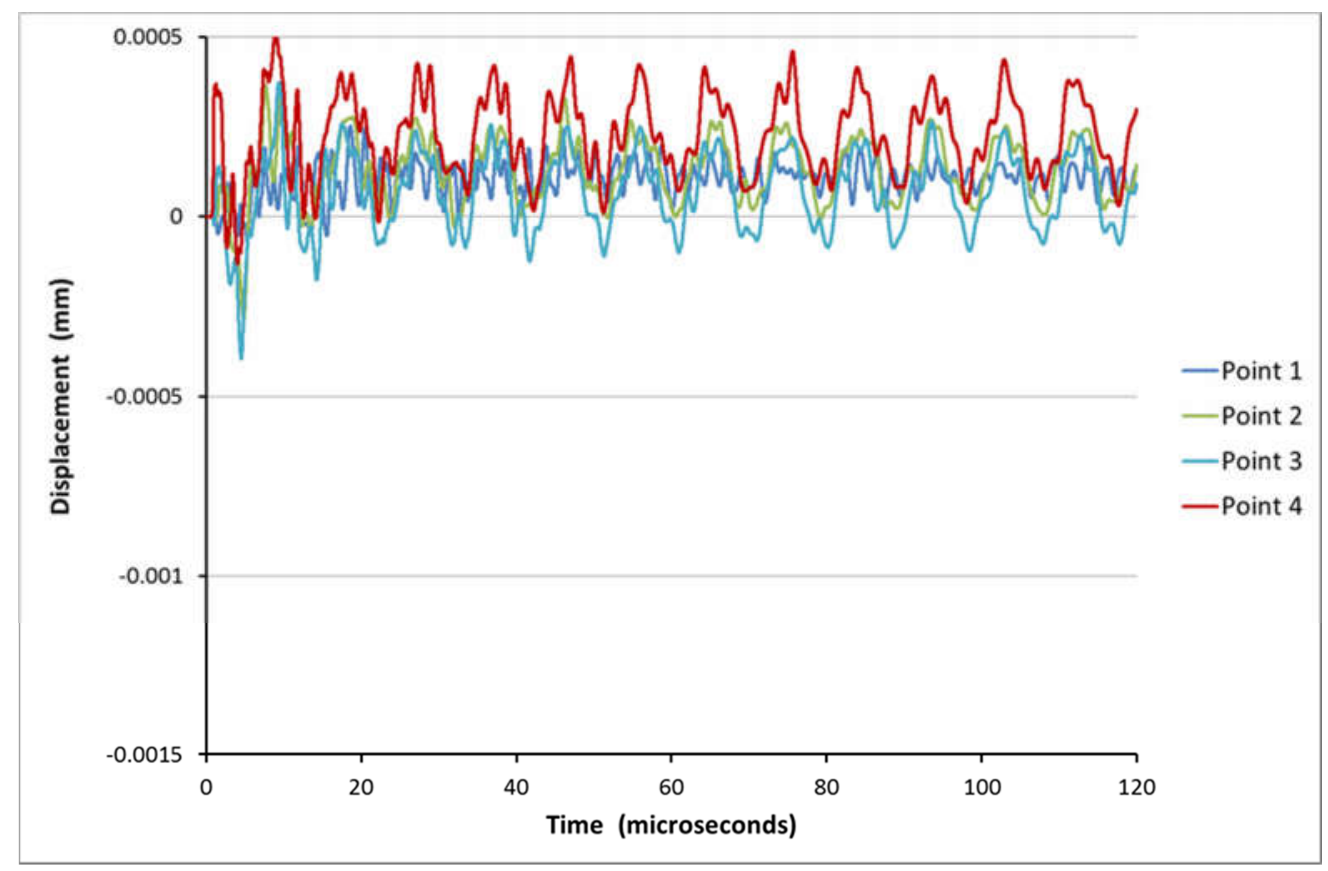

Figure 25 Lateral displacements of nodes lying along the mid-plane of the specimen for the optimised configuration 
Figure 26 shows comparison between the baseline and the new optimised configuration for strain in the $\mathrm{z}$ direction (impact direction) in the specimen. The states (a), (b), (c), (d) show the propagation of compressive and corresponding release waves for the specimen. State (e) shows a more uniform unloading of the specimen by the release wave originated from the flyer plate free surface. State (f) shows the final through thickness $(Z)$ strain distribution indicating that there is no secondary impact the strain field in the new configuration is much more uniform than in the baseline configuration.

Comparison of the level of the strain in $\mathrm{x}$ direction (radial direction) between the baseline and new configuration is shown in Figure 27. It can be clearly seen that this strain in the new configuration is significantly reduced. In particular in state (f) the final radial strain field in the new configuration is more uniform than in the baseline configuration since the specimen did not suffer any secondary impacts.

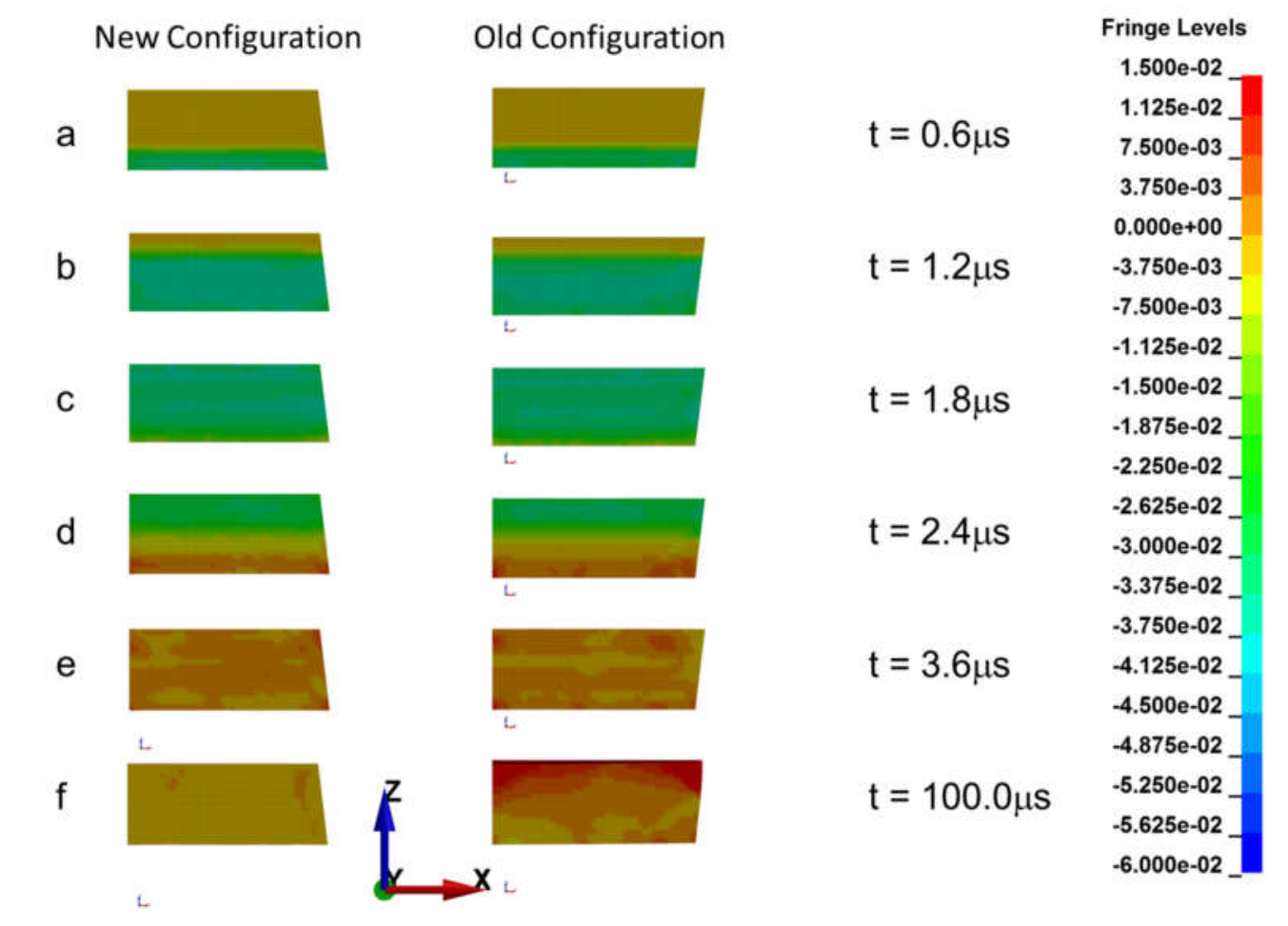

Figure 26 - Comparison between optimised (left) and baseline (right) Specimen strains in the impact $(Z)$ direction as a function of time $(\mu \mathrm{s})$ 


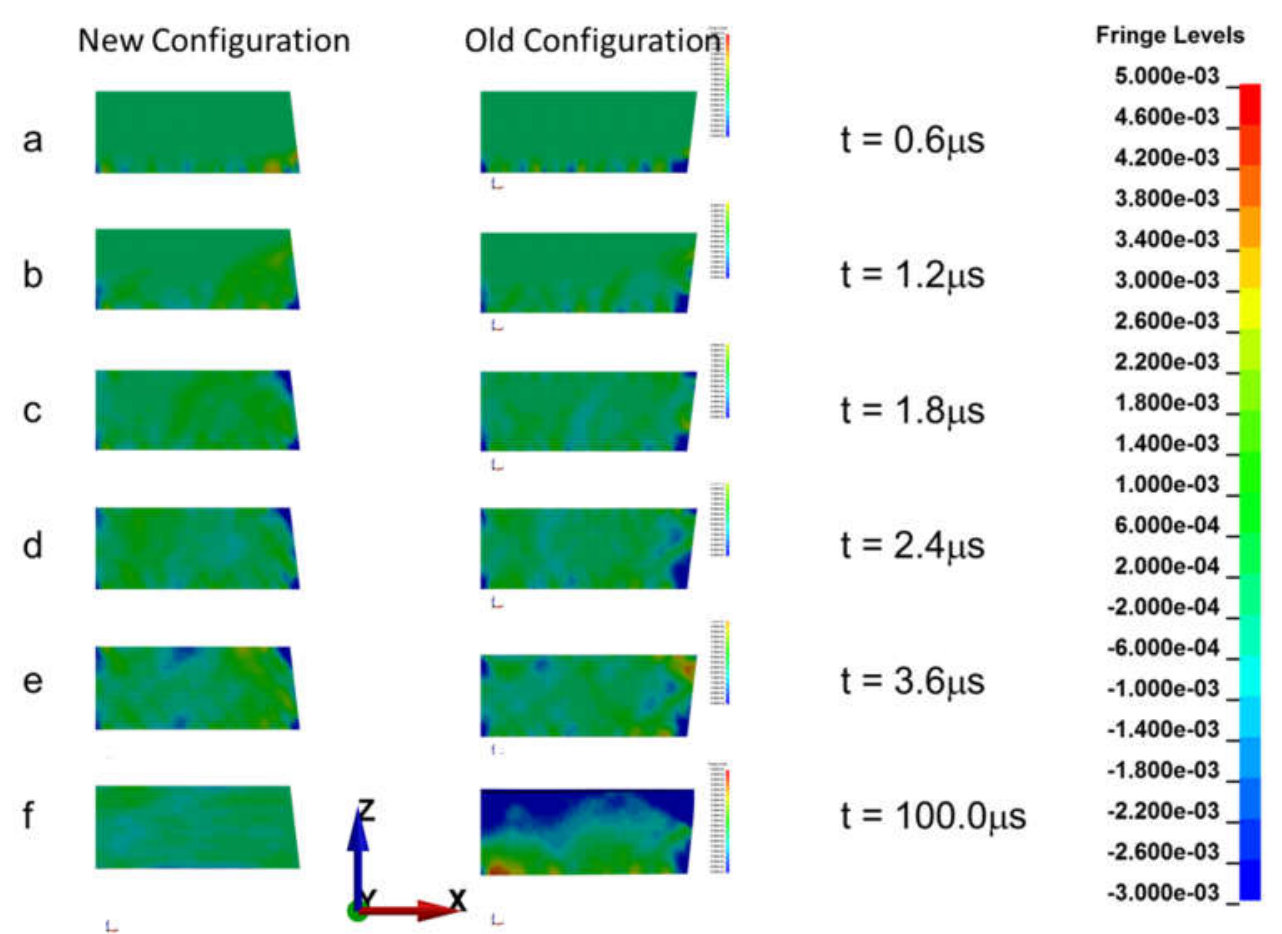

Figure 27 - Comparison between optimised (left) and baseline (right) Specimen strains in the through thickness $(Z)$ direction as a function of time ( $\mu \mathrm{s})$

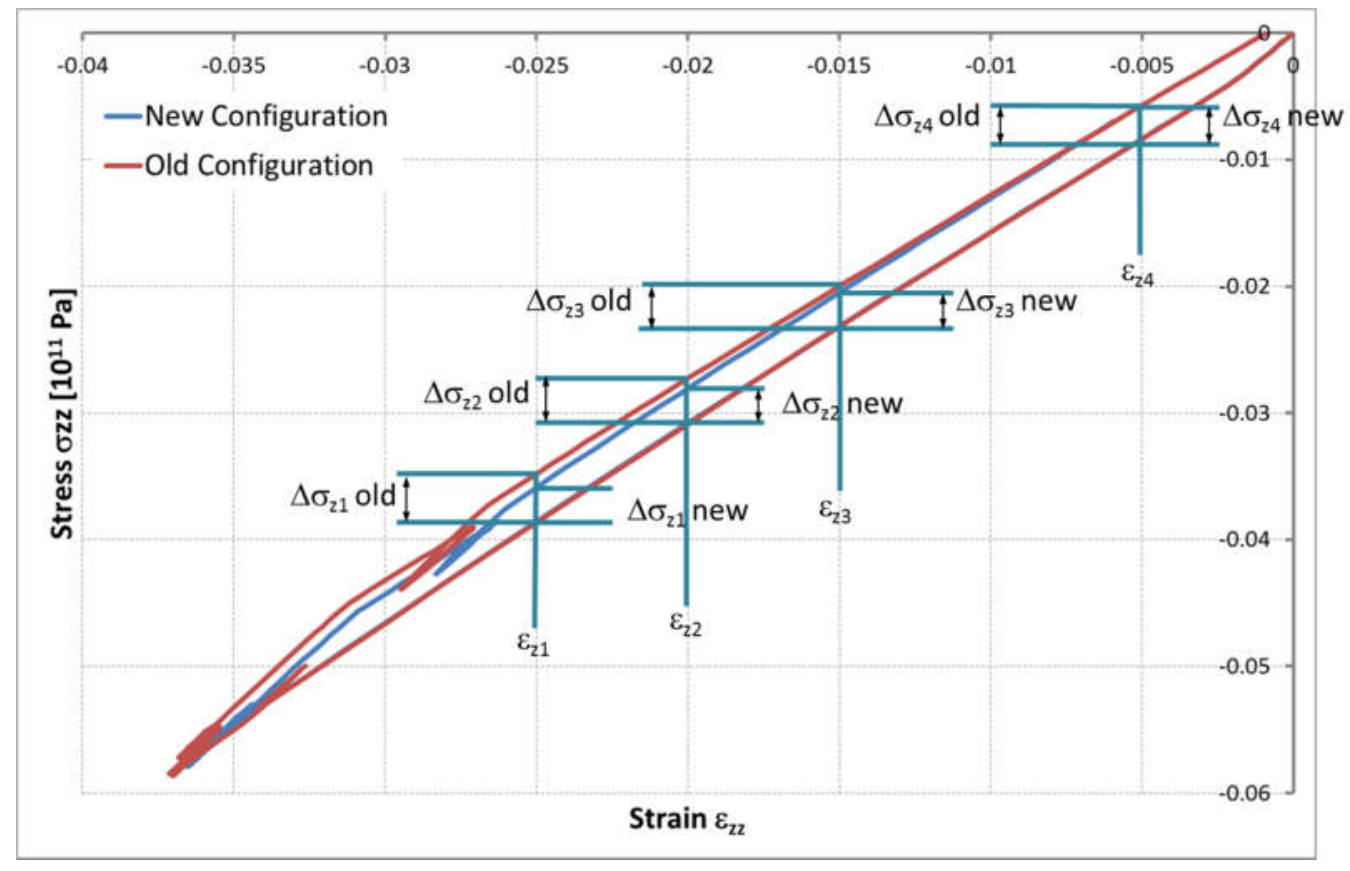

Figure 28 Stress-Strain curve in the through thickness $(Z)$ direction, showing the loading and unloading path for a central element in the specimen for baseline and optimised configurations 
Figure 28 provides a comparison between stress-strain curve for the baseline and optimised configurations. As can be seen, the loading path for both configurations is the same. The differences in the magnitudes in the stresses have been compared along the unloading part of curve only and the results can be found in Table 3 .

Table 1: Difference between the loading and unloading path; initial and final configuration

\begin{tabular}{|c|c|c|c|c|}
\hline & $\begin{array}{c}\varepsilon_{\mathrm{z} 1}=-0.025 \\
\Delta \sigma_{\mathrm{z} 1}\left(10^{11} \mathrm{~Pa}\right)\end{array}$ & $\begin{array}{c}\varepsilon_{\mathrm{z} 2}=-0.02 \\
\Delta \sigma_{\mathrm{z} 2}\left(10^{11} \mathrm{~Pa}\right)\end{array}$ & $\begin{array}{c}\varepsilon_{\mathrm{z} 3}=-0.015 \\
\Delta \sigma_{\mathrm{z} 3}\left(10^{11} \mathrm{~Pa}\right)\end{array}$ & $\begin{array}{c}\varepsilon_{\mathrm{z} 4}=-0.005 \\
\Delta \sigma_{\mathrm{z} 4}\left(10^{11} \mathrm{~Pa}\right)\end{array}$ \\
\hline Old & 0.00386 & 0.00359 & 0.00319 & 0.00263 \\
\hline New & 0.00272 & 0.00276 & 0.00268 & 0.00282 \\
\hline Time $(\mu \mathrm{s})$ & 2.21 & 2.28 & 2.34 & 2.45 \\
\hline
\end{tabular}

The results presented in Table 3 show that the unloading part of the curve for the optimised setup is more parallel with the loading path, i.e. closer to the state of uniaxial strain. This clearly shows an improvement of the optimised configuration. In the beginning of the unloading, the baseline configuration has the same value of $\Delta \sigma_{z}$ as the optimised configuration.

Figure 29 shows the rigid body velocities vs. time for the cover plate, specimen and spall plate. From these results, it can be seen that specimen velocity is reduced compared to baseline, with a reduction from $17.8 \mathrm{~ms}^{-1}$ to $14.6 \mathrm{~ms}^{-1}$, representing a $18 \%$ reduction. However due to the secondary impact the velocity in the baseline configuration increases to $23.6 \mathrm{~ms}^{-1}$, which then represents a $38 \%$ reduction. 


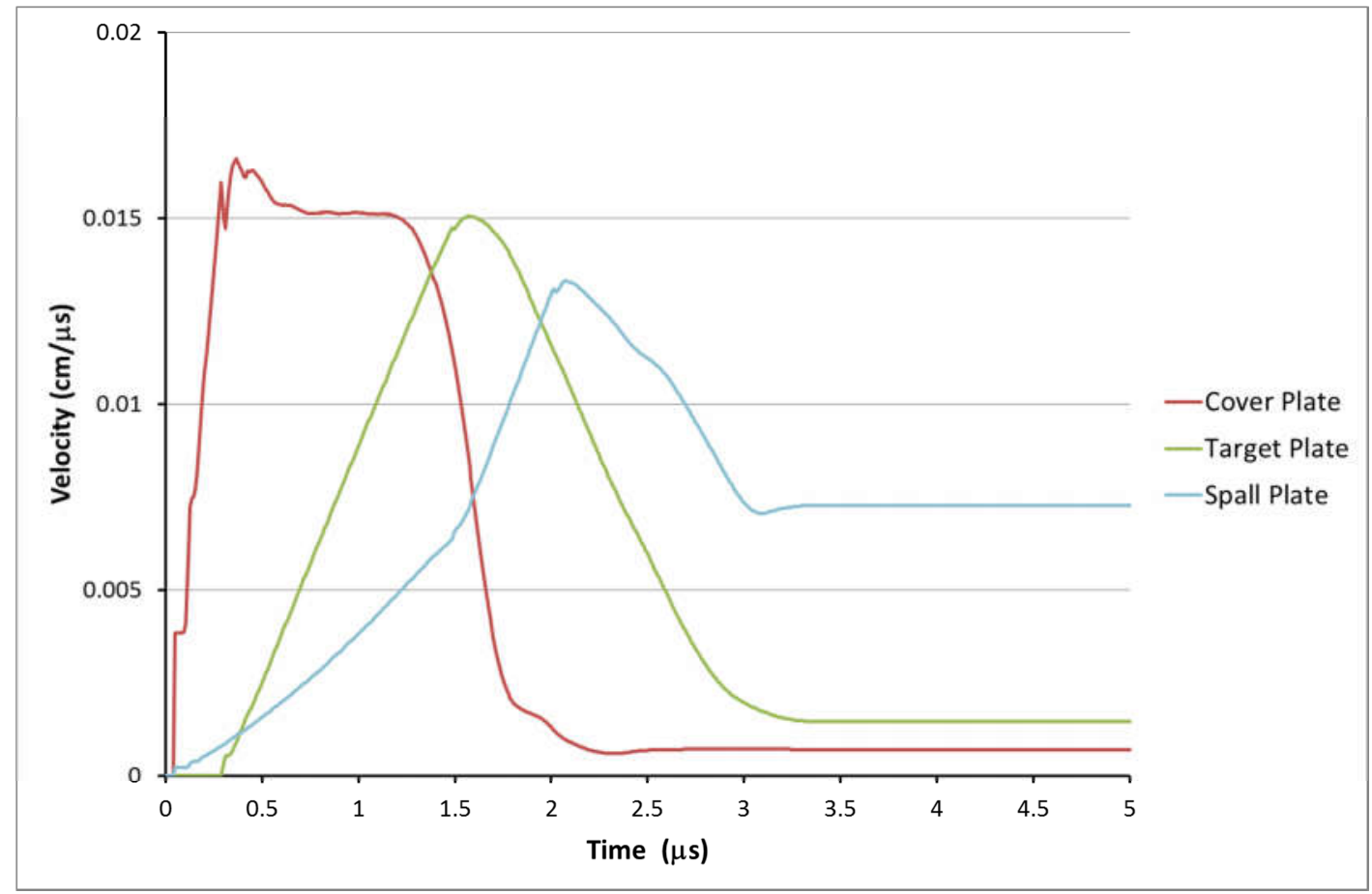

Figure 29 -Velocity-time diagram for the cover plate, specimen and spall plate for the optimised configuration

The conclusion from this work shows that changing the geometry of the baseline configuration has a positive influence on the results obtained for the specimen. The secondary impact between the target plate and inner momentum trap ring was eliminated by modifying the design. The results show that the radial release and residual velocity for the target were reduced. Changing geometry has the potential to further decrease the residual velocity, but would also influence the formation of radial release waves.

\section{CONCLUSION}

The objective of this research was to develop and optimise a new test configuration, based on the investigation of a baseline set up and identification of the key design parameters that affect performance of the shock recovery assembly. 
Through the use of non-linear transient finite element analysis shortcomings of the baseline configuration were identified, including the impact of the inner ring (inner lateral momentum trap) with the specimen, interaction of two longitudinal release waves spread over a number of spall plates.

Based on this information, an alternative configuration was investigated, in order to remove these unwanted effects. The analysis of the new configuration showed a number of improvements. Comparing distance-time diagrams, it was found that longitudinal wave reflection was decreased and through reducing the number of spall plates from four to three in a $2-1-1 \mathrm{~mm}$ configuration, resulted in the longer release wave interaction within the $2 \mathrm{~mm}$ spall plate. Through investigating the loadingunloading path, and lateral displacements at various points within the specimen, it was shown that the influence of radial release was decreased.

The new configuration resulted in the decrease of both the velocity and radial deformation of the specimen. The secondary impact of the specimen and inner ring was eliminated and the final residual velocity was decreased by $38 \%$ compared to baseline and the maximum displacement in the radial direction was decreased by $27 \%$.

\section{REFERENCES}

[1] T. Nicholas and A.M. Rajendran. Material Characterisation at High Strain Rates. In: J.A. Zukas (Ed.) High Velocity Impact Dynamics. John Wiley \& Sons, 1990.

[2] M. A. Meyers and L. E. Murr, Shock Waves and High Strain Rate phenomenon in metals, Editors M. A. Meyers and L. E. Murr, Plenum Press, N.Y, 1981: pp.341-373 
[3] J.E. Field, S.M. Walley, W.G. Proud, H.T. Goldrein, C.R. Siviour, Review of experimental techniques for high rate deformation and shock studies, International Journal of Impact Engineering, 30 (2004) 725-775.

[4] G. Raiser, R.J. Clifton and M.Ortiz. A soft-recovery plate impact experiment for studying microcracking in ceramics. Mechanics of Materials 10 (1990) 4358.

[5] F. Llorca, F. Buy and J. Farre Experimental analysis of shock wave effects in copper. In Shock compression of condensed matter-2001 (ed. M. D. Furnish, N. N. Thadhani \& Y. Horie), (2002) 638-641. Melville, NY: American Institute of Physics

[6] T. d'Almeida, D.J. Chapman, W.G. Proud, P.J. Gould, P.D. Church, M. Reynolds, R. Wheeler, H.J. MacGillivray, M. Di Michiel and J.M. Merino. Soft recovery of a ferrous alloy: Structural modification and properties. In: Proceedings of DYMAT2009, 971-976 (2009), DOI: 10.1051/dymat/2009136

[7] N.K. Bourne, G.T. Gray III and J.C.F. Millett. On the shock response of cubic metals. J. Appl. Phys. 106, 091301 (2009); doi: 10.1063/1.321875

[8] R. L. Rabie, J. E. Vorthman and J. K. Dienes, Shock Waves in Condensed Matter 1983. Proceedings of the American Physical Society Topical Conference. 1984: 199-202

[9] D. G. Doran and R. K. Linde, Shock effects in solids. Solid State Physics, 19 (1966), 229-290.

[10] A. L. Stevens and O. E. Jones. Radial stress release phenomena in plate impact experiments - compression-release Journal of Applied Mechanics, 39 (1972) $359-366$ 
[11] N.K. Bourne and G.T. Gray III. Computational design of recovery experiments for ductile metals. Proc. R. Soc. A 461 (2005) doi: 10.1098/rspa.2005.1501

[12] M.D. Furnish, G.T. Gray III, J.F. Bingert. Line VISAR and Post-Shot Metallography Comparisons for Spall Analysis, Proceedings of the SEM Annual Conference, June 7-10, 2010, Indianapolis, Indiana, USA.

[13] T.J. Vogler, J.D. Clayton. Heterogeneous deformation and spall of an extruded tungsten alloy: plate impact experiments and crystal plasticity modeling, Journal of thye Mechanics and Physics of Solids, 56 (2008) 297-335.

[14] T.J Vogler, et al. Using the line-VISAR to study multi-dimensional and mesoscale impact phenomena, International Journal of Impact Engineering, 35 (2008) 1844-1852.

[15] M. Stojkovic, Shock Recovery Experiment, Thesis (2003) Cranfield University, Cranfield, UK. 